\title{
Prospects for vectorlike leptons at future proton-proton colliders
}

\author{
Prudhvi N. Bhattiprolu and Stephen P. Martin \\ Department of Physics, Northern Illinois University, DeKalb, Illinois 60115, USA
}

(Received 8 May 2019; published 19 July 2019)

\begin{abstract}
Vectorlike leptons are an intriguing possibility for physics beyond the Standard Model. We study the reach for discovering (at $5 \sigma$ significance) or excluding (at $95 \%$ confidence) models of charged vectorlike leptons that mix predominantly with the tau, using multilepton signatures at various future proton-proton collider options: a high-luminosity LHC with $\sqrt{s}=14 \mathrm{TeV}$, a high-energy LHC with $\sqrt{s}=27 \mathrm{TeV}$, and possible new longer-tunnel colliders with $\sqrt{s}=70$ or $100 \mathrm{TeV}$. For weak-isodoublet vectorlike leptons, we estimate that a $27 \mathrm{TeV}$ high-energy LHC with $15 \mathrm{ab}^{-1}$ could exclude masses up to about $2300 \mathrm{GeV}$, or discover them if the mass is less than about $1700 \mathrm{GeV}$, while a $100 \mathrm{TeV}$ collider with $30 \mathrm{ab}^{-1}$ could exclude masses up to about $5750 \mathrm{GeV}$ or make a discovery if the mass is less than about $4000 \mathrm{GeV}$. However, we find that weak-isosinglet vectorlike leptons present a much more difficult challenge, with some reach for exclusion, but not for discovery at any of the collider options considered.
\end{abstract}

DOI: 10.1103/PhysRevD.100.015033

\section{INTRODUCTION}

The Large Hadron Collider (LHC) has conducted searches for new physics beyond the Standard Model, at proton-proton center of mass energies up to $\sqrt{s}=13 \mathrm{TeV}$. Following the discovery [1] of the Higgs scalar boson with mass near $M_{h}=125 \mathrm{GeV}$, the present evidence from the LHC is consistent with the minimal version of the Standard Model, with direct and indirect limits on new particles extending well into the $\mathrm{TeV}$ range. However, there are many candidate new physics models that obey decoupling as the mass scale of new physics is raised. These include supersymmetric theories, where agreement with the Standard Model gets better as the scale of supersymmetry breaking is raised. An example of a nondecoupling theory is a new chiral fourth family of fermions. Because of the necessity of generating the large chiral fermion masses through Yukawa couplings and the Higgs mechanism, decoupling is violated, which causes significant contributions to precision electroweak observables as well as Higgs boson production and decay rates, in conflict [2] with the observations.

New fermions are still allowed, because they will obey decoupling, if they obtain their mass entirely or mostly as bare electroweak-singlet terms in the Lagrangian, rather than from the Yukawa couplings to the Higgs field. This implies that the new fermions are vectorlike (the antonym

Published by the American Physical Society under the terms of the Creative Commons Attribution 4.0 International license. Further distribution of this work must maintain attribution to the author(s) and the published article's title, journal citation, and DOI. Funded by SCOAP. of chiral). It is a notable common feature of many new physics theories that are motivated for other reasons (such as the need for a cosmological and astrophysical dark matter candidate, or to address the hierarchy problem) that they often contain vectorlike fermions. For example, in supersymmetry [3], the Higgsinos have the same gauge quantum numbers as would a vectorlike pair of weakisodoublet leptons. It is plausible that additional vectorlike fermion masses are at the $\mathrm{TeV}$ scale, because whatever mechanism is responsible for the Higgsino masses can also be applied to the vectorlike fermion masses, which have the same structure. Vectorlike fermions can therefore be added on to the minimal version of supersymmetry, with one possible benefit that they can contribute to the lightest Higgs boson mass [4-18], which allows a lower supersymmetry breaking scale consistent with $M_{h}=125 \mathrm{GeV}$. For example, adding vectorlike quarks to the simplest models of gauge-mediated supersymmetry breaking allows [15-18] them to be still discoverable at the LHC. However, even ignoring such motivations, it is also worthwhile to consider vectorlike fermions merely on the merits of being a simple and consistent candidate extension of the Standard Model.

For vectorlike quarks, the LHC pair production cross section is determined from QCD and is therefore large and essentially model independent. ${ }^{1}$ The searches for vectorlike quarks are consequently quite advanced, with current LHC limits (depending on the specific decay modes) on

\footnotetext{
${ }^{1}$ Single production of vectorlike quarks is also subject to LHC limits, but in a much more model-dependent way.
} 
pair-produced vectorlike quarks that can be found in Refs. [19,20].

In contrast, vectorlike leptons (VLLs) [21-71] are pairproduced by $s$-channel electroweak vector boson diagrams, leading to cross sections that are much smaller and dependent on the choices of weak isospin and weak hypercharge of the new states. ${ }^{2}$ For this reason, until recently the LHC detector collaborations had not put limits on VLLs beyond those that could be inferred from the CERN large electron-positron (LEP) $e^{-} e^{+}$collider, which excluded [74] masses up to $101.2 \mathrm{GeV}$. There have been several phenomenological studies that looked into the LHC discovery and exclusion capabilities. In Ref. [50], search strategies and capabilities were discussed for the optimistic case that the VLLs decayed mostly to muons, and an ATLAS search was conducted for that case in [52]. In Ref. [57], the more pessimistic case of decays to tau-rich final states was considered, and it was argued that even with the (then current, but now old) data at $\sqrt{s}=8 \mathrm{TeV}$ it should be possible to exclude such $S U(2)_{L}$-doublet VLLs up to about $M_{\tau^{\prime}}=275 \mathrm{GeV}$, but not $S U(2)_{L^{-}}$-singlet charged VLLs, which have a much smaller production cross section and unfavorable branching ratios. Since then, the CMS collaboration has published [70,71] the results of dedicated searches for doublet VLLs, based on 41.4 and $77.4 \mathrm{fb}^{-1}$ data samples at $\sqrt{s}=13 \mathrm{TeV}$. Aided in part by a deficit of events in the signal regions compared to the background expectation, CMS was able to obtain an exclusion for $120<M_{\tau^{\prime}}<790 \mathrm{GeV}$ for a pure $S U(2)_{L^{-}}$ doublet VLL pair that mixes with and decays to tau leptons.

In this paper, we will consider the prospects for exclusion or discovery of VLLs at planned future proton-proton colliders. While there are a wide variety of possible VLL models, we will consider as benchmark models two simple minimal cases as defined in Ref. [57], a "singlet VLL" and a "doublet VLL" model, with the mixing to the Standard Model leptons assumed to be entirely with the tau and small. One advantage of these models as benchmarks is that the pair-production cross sections are uniquely determined as a function of the mass, as discussed in Ref. [57] and reviewed in the next section. As was the case in Ref. [57], our analysis below shows that the minimal singlet VLL model is very difficult to probe at proton-proton colliders. We will therefore also consider a class of nonminimal models which are similar to the minimal singlet VLL model and have the same production cross section, but have different branching ratios for the lightest charged new lepton.

We will consider the following proton-proton collider options:

\footnotetext{
${ }^{2}$ Here we concentrate on models with new charged vectorlike leptons. Signatures from models with neutrino masses from electroweak-singlet states at the $\mathrm{TeV}$ scale are reviewed in $[72,73]$, for example.
}

(i) a high-luminosity LHC (HL-LHC), defined as $3 \mathrm{ab}^{-1}$ of proton-proton collisions at $\sqrt{s}=14 \mathrm{TeV}$,

(ii) a high-energy LHC (HE-LHC), defined as $15 \mathrm{ab}^{-1}$ at $\sqrt{s}=27 \mathrm{TeV}$,

(iii) a very high-energy $p p$ collider, defined as up to $30 \mathrm{ab}^{-1}$ at either $\sqrt{s}=70$ or $100 \mathrm{TeV}$, which could be realized as, e.g., a CERN hadron-hadron future circular collider [75] or a super proton-proton collider [76] in China.

The physics potential for HL-LHC and HE-LHC has been studied for various other beyond-the-Standard-Model scenarios in Ref. [77] and references therein.

For reach estimates, we will use a simple cut-based counting experiment criteria, where the significance

$$
Z=\sqrt{2} \operatorname{erfc}^{-1}(2 p)
$$

of an experimental result is related to the probability $p$ of observing, in an ensemble of many repeated experiments, a result of equal or greater incompatibility with the null hypothesis. Let the number of background events be Poisson distributed with mean $b$, with the variance in $b$ (corresponding intuitively to an uncertainty in the expected number of background events) given by $\Delta_{b}$, and suppose the number of signal events is Poisson distributed with predicted mean $s$. Then the median expected significance for discovery (where the null hypothesis is background only, and the signal is assumed to be present in the data) is approximately [78-81]

$$
\begin{aligned}
Z_{\mathrm{disc}}= & {\left[2 \left((s+b) \ln \left[\frac{(s+b)\left(b+\Delta_{b}^{2}\right)}{b^{2}+(s+b) \Delta_{b}^{2}}\right]\right.\right.} \\
& \left.\left.-\frac{b^{2}}{\Delta_{b}^{2}} \ln \left[1+\frac{s \Delta_{b}^{2}}{b\left(b+\Delta_{b}^{2}\right)}\right]\right)\right]^{1 / 2} .
\end{aligned}
$$

The median expected significance for exclusion (where the null hypothesis is now the background + signal model, but the signal is assumed to be actually absent in the data) can be approximated by [57]

$$
\begin{aligned}
Z_{\mathrm{exc}}= & \left\{2\left[s-b \ln \left(\frac{b+s+x}{2 b}\right)-\frac{b^{2}}{\Delta_{b}^{2}} \ln \left(\frac{b-s+x}{2 b}\right)\right]\right. \\
& \left.-(b+s-x)\left(1+b / \Delta_{b}^{2}\right)\right\}^{1 / 2},
\end{aligned}
$$

where

$$
x=\sqrt{(s+b)^{2}-4 s \Delta_{b}^{2} /\left(1+\Delta_{b}^{2} / b\right)} .
$$

In the idealized limit of a perfectly known background prediction, $\Delta_{b}=0$, these would reduce to

$$
Z_{\mathrm{disc}}=\sqrt{2[(s+b) \ln (1+s / b)-s]},
$$




$$
Z_{\mathrm{exc}}=\sqrt{2[s-b \ln (1+s / b)]} .
$$

In the further limit of large $b$, these both approach $Z_{\text {disc }}=$ $Z_{\text {exc }}=s / \sqrt{b}$. In the following, we will use as a criteria for expected five-sigma discovery that $s$ is at least 10 and Eq. (1.2) should exceed $Z_{\text {disc }}>5$, and for an expected $95 \%$ exclusion that Eq. (1.3) should exceed $Z_{\text {exc }}>1.645$ corresponding to $p=0.05$. We will use the somewhat arbitrary (since the capabilities of the detectors are unknown at present) choices $\Delta_{b} / b=0.1,0.2$, and 0.5 , corresponding to a $10 \%, 20 \%$, and $50 \%$ uncertainty in the background. We have also assumed that $b$ is always at least one event, to be conservative.

\section{MINIMAL MODELS FOR PRODUCTION AND DECAY OF VECTORLIKE LEPTONS}

Following the terminology and definitions in Ref. [57], the singlet VLL model contains the Standard Model fields and interactions plus an $S U(2)_{L}$-singlet charged VLL $\tau^{\prime-}$ and its antiparticle $\tau^{\prime+}$, which consist of twocomponent left-handed fermions transforming under $S U(3)_{c} \times S U(2)_{L} \times U(1)_{Y}$ as

$$
\tau^{\prime}+\bar{\tau}^{\prime}=(\mathbf{1}, \mathbf{1},-1)+(\mathbf{1}, \mathbf{1},+1) .
$$

The doublet VLL model contains a new charged lepton and its neutral heavy Dirac neutrino partner, realized as twocomponent left-handed fermions transforming as

$$
\begin{aligned}
L+\bar{L} & =\left(\begin{array}{c}
\nu^{\prime} \\
\tau^{\prime}
\end{array}\right)+\left(\begin{array}{c}
\bar{\tau}^{\prime} \\
\bar{\nu}^{\prime}
\end{array}\right) \\
& =\left(\mathbf{1}, 2,-\frac{1}{2}\right)+\left(\mathbf{1}, 2,+\frac{1}{2}\right) .
\end{aligned}
$$

In both models, a single weak-isosinglet bare fermion mass parameter $M$ is mostly responsible for the new fermion masses, with a small Yukawa couplings $\epsilon$ to the Higgs field providing the mixing mass with the Standard Model $\tau$ lepton, which also has its own Yukawa coupling $y_{\tau}$ to the Higgs field. The charged fermion mass matrix for the $\tau, \tau^{\prime}$ system in each case then can be written in the form

$$
\mathcal{M}=\left(\begin{array}{cc}
y_{\tau} v & 0 \\
\epsilon v & M
\end{array}\right)
$$

where $v \approx 174 \mathrm{GeV}$ is the Standard Model Higgs vacuum expectation value. The tree-level mass eigenvalues, obtained from the square roots of the eigenvalues of $\mathcal{M}^{\dagger} \mathcal{M}$ after expanding for $y_{\tau} v, \epsilon v \ll M$, are

$$
\begin{aligned}
& M_{\tau}=y_{\tau} v\left(1-\epsilon^{2} v^{2} / 2 M^{2}+\cdots\right), \\
& M_{\tau^{\prime}}=M\left(1+\epsilon^{2} v^{2} / 2 M^{2}+\cdots\right),
\end{aligned}
$$

where the ellipses in both cases represent terms suppressed by $\epsilon^{4} v^{4} / M^{4}$ or $\epsilon^{2} y_{\tau}^{2} v^{4} / M^{4}$, while the tree-level neutral VLL mass in the doublet model is simply $M_{\nu^{\prime}}=M$. There is also a one-loop radiative correction to the physical mass splitting $M_{\nu^{\prime}}-M_{\tau^{\prime}}$, but it is also small, only of order of a few hundred $\mathrm{MeV}$ [23].

In the special case of no mixing with the tau lepton, $\epsilon=0$, the lightest VLL would be absolutely stable due to a conserved global symmetry. Here, we assume instead that $\epsilon$ is small enough to be treated as a tiny perturbation in the mass matrix, but that it exceeds about $2 \times 10^{-7}$, which is large enough to allow [57] the VLLs to decay promptly ${ }^{3}$ on collider detector length scales to Standard Model states, with widths that dominate over the competing mode $\tau^{-\prime} \rightarrow \pi^{-} \nu^{\prime}$ [23] in the doublet VLL case. The fermion mass eigenstates then consist of, besides the usual $\tau^{+}, \tau^{-}, \nu_{\tau}$ and the rest of the Standard Model states, a charged Dirac pair $\tau^{\prime-}, \tau^{\prime+}$ in both models, and a neutral Dirac pair $\nu^{\prime}, \bar{\nu}^{\prime}$ in the doublet VLL model only. Due to the small size of $\epsilon$ and the one-loop radiative splitting, we can take $M_{\nu^{\prime}} \approx M_{\tau^{\prime}}$ for the purposes of collider simulations.

The collider pair-production modes are

$$
\begin{gathered}
p p \rightarrow \gamma^{*}, \quad Z^{*} \rightarrow \tau^{\prime-} \tau^{\prime+}, \\
p p \rightarrow Z^{*} \rightarrow \nu^{\prime} \bar{\nu}^{\prime}, \\
p p \rightarrow W^{-*} \rightarrow \tau^{\prime-} \bar{\nu}^{\prime}, \\
p p \rightarrow W^{+*} \rightarrow \tau^{\prime+} \nu^{\prime}
\end{gathered}
$$

involving couplings to the $\gamma, Z$, and $W^{ \pm}$vector bosons, which are listed in Ref. [57]. Of course, only the first of these processes occurs for the singlet VLL model.

In Fig. 1, we show the total pair-production cross sections at a proton-proton collider as a function of $M_{\tau^{\prime}}=M_{\nu^{\prime}}$, for the choices $\sqrt{s}=8,13,14,27,70,100 \mathrm{TeV}$. Note that the doublet VLL model has a considerably larger total cross section, which is partly responsible for the much better search prospects as found in Ref. [57] and below in the present paper. For example, at $M_{\tau^{\prime}}=1000 \mathrm{GeV}$, the total cross section is about 12.5 times larger in the doublet VLL model than for the singlet VLL model, nearly independent of the proton-proton collision energy over the range from 14 to $100 \mathrm{TeV}$. In the doublet VLL model, the total contribution is dominated by the $W$-boson-mediated $\tau^{\prime-} \bar{\nu}^{\prime}$ and $\tau^{\prime+} \nu^{\prime}$ final states. However, even if one restricts attention to only the $\tau^{\prime-} \tau^{\prime+}$ final state common to both models, the doublet

\footnotetext{
${ }^{3}$ If the $\tau^{\prime}$ in the singlet VLL model is quasistable, then search strategies based on time of flight and ionization rate should apply, as in the ATLAS search in Ref. [82]. Assuming that the $\tau^{\prime}$ interacts similar to a chargino, we infer from the cross section limit in Fig. 10 of [82] that its mass should exceed about $750 \mathrm{GeV}$. This does not apply to the doublet VLL model, where the $\tau^{\prime}$ can decay to $\nu^{\prime}$ inside the detector.
} 

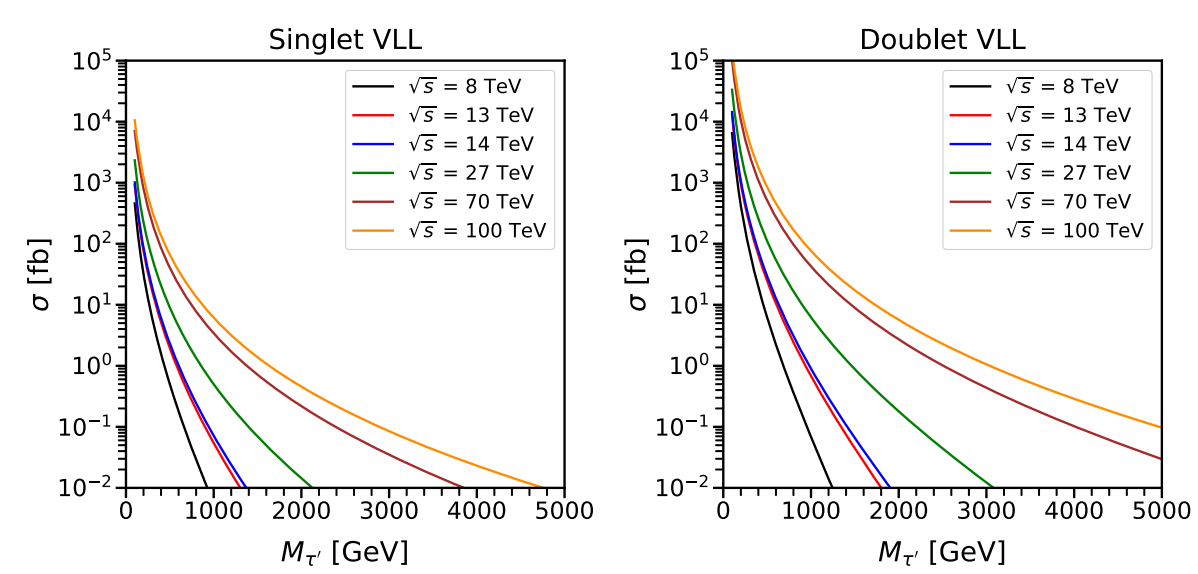

FIG. 1. The total pair-production cross sections for $\tau^{\prime-} \tau^{\prime+}$ in the singlet VLL model (left) and for the sum of $\tau^{\prime-} \tau^{\prime+}$ and $\nu^{\prime} \bar{\nu}^{\prime}$ and $\tau^{\prime-} \bar{\nu}^{\prime}$ and $\tau^{\prime+} \nu^{\prime}$ in the doublet VLL model (right), for proton-proton collisions at $\sqrt{s}=8,13,14,27,70,100 \mathrm{TeV}$ (bottom to top).

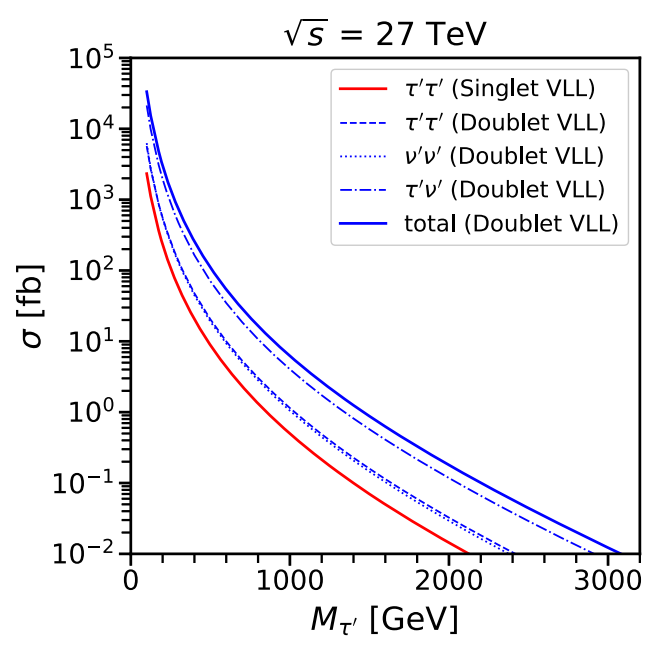

FIG. 2. The individual pair-production cross sections in the singlet VLL model for $\tau^{\prime-} \tau^{\prime+}$ (solid red line) and in the doublet VLL model for $\tau^{\prime-} \tau^{\prime+}$ (dashed blue line) and $\nu^{\prime} \bar{\nu}^{\prime}$ (dotted blue line) and $\tau^{\prime-} \bar{\nu}^{\prime}$ plus $\tau^{\prime+} \nu^{\prime}$ (dot-dashed blue line) and total (solid blue line), for proton-proton collisions at $\sqrt{s}=27 \mathrm{TeV}$.

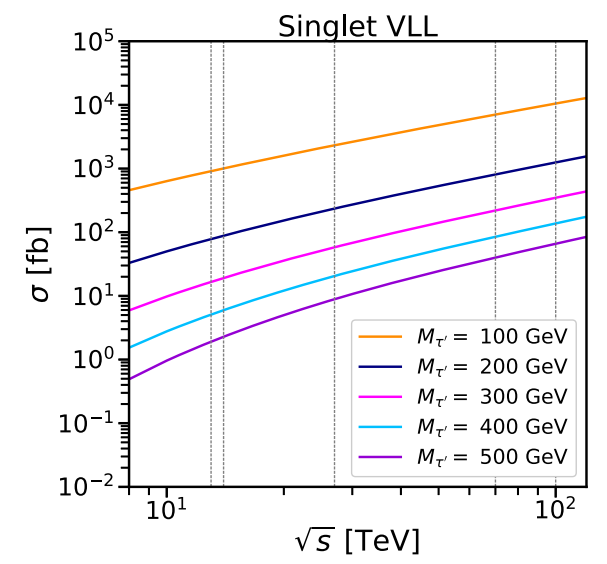

VLL model has a significantly larger cross section. This is illustrated in Fig. 2, which compares the individual cross sections for each of the final states at $\sqrt{s}=27 \mathrm{TeV}$. (Results are similar at other values of $\sqrt{s}$.)

The total pair-production cross sections for these models are also shown in Fig. 3 as a function of $\sqrt{s}$, for various choices of $M_{\tau^{\prime}}=M_{\nu^{\prime}}$. Clearly, the cross sections are getting larger at higher collider energies. In the doublet VLL model, one obtains roughly the same cross section for $M_{\tau^{\prime}}=4000 \mathrm{GeV}$ at $\sqrt{s}=100 \mathrm{TeV}$ as one would have for $M_{\tau^{\prime}}=1870 \mathrm{GeV}$ at $\sqrt{s}=27 \mathrm{TeV}$ or for $M_{\tau^{\prime}}=1220 \mathrm{GeV}$ at $\sqrt{s}=14 \mathrm{TeV}$.

As for the decays, the lepton-flavor conserving charged current process $\tau^{\prime} \rightarrow \nu^{\prime} \pi^{-}$is kinematically allowed in the doublet VLL model, but has a width smaller than the direct lepton-flavor-violating decays to Standard Model fermions as long as $\epsilon \gtrsim 2 \times 10^{-7}$ [57] and is therefore neglected. In the doublet VLL model, the neutral VLLs decay $100 \%$ of the time to a $W$ boson and an ordinary tau lepton, with branching ratios $(\mathrm{BR})$

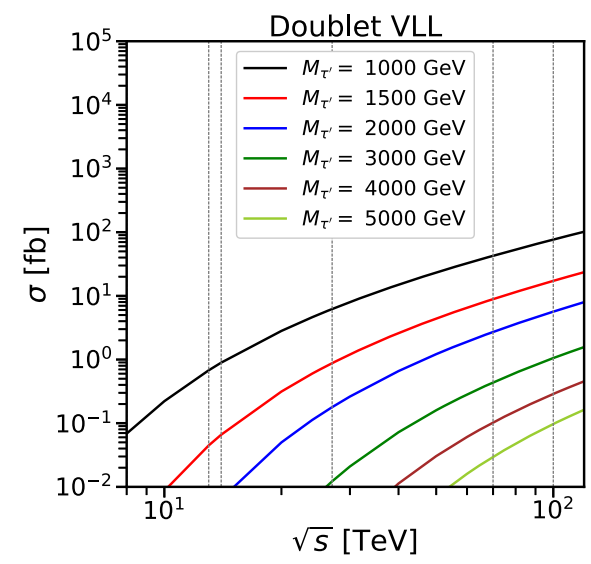

FIG. 3. The total pair-production cross sections for $\tau^{\prime-} \tau^{\prime+}$ in the singlet VLL model (left) and for the sum of $\tau^{\prime-} \tau^{\prime+}$ and $\nu^{\prime} \bar{\nu}^{\prime}$ and $\tau^{\prime-} \bar{\nu}^{\prime}$ and $\tau^{\prime+} \nu^{\prime}$ in the doublet VLL model (right), as a function of $\sqrt{s}$, for various values of $M_{\tau^{\prime}}=M_{\nu^{\prime}}$ as labeled. 


$$
\mathrm{BR}\left(\nu^{\prime} \rightarrow W^{+} \tau^{-}\right)=\mathrm{BR}\left(\bar{\nu}^{\prime} \rightarrow W^{-} \tau^{+}\right)=1
$$

This reflects our assumptions that there is no $\nu^{\prime}$ mixing with the left-handed neutrinos of the Standard Model and that the mixing Yukawa coupling $\epsilon$ involves the tau lepton, rather than muon or the electron; otherwise the discovery and exclusion strategies would be much easier, due to the higher detection efficiency and lower fake rates for $e, \mu$ compared to $\tau$.

In both models, the charged VLLs can decay into the final states $Z \tau, h \tau$, and $W \nu_{\tau}$. The decay widths (neglecting the tau lepton mass) are [57]

$$
\begin{gathered}
\Gamma\left(\tau^{\prime} \rightarrow h \tau\right)=\frac{\epsilon^{2}}{64 \pi} M_{\tau^{\prime}}\left(1-r_{h}\right)^{2}, \\
\Gamma\left(\tau^{\prime} \rightarrow Z \tau\right)=\frac{\epsilon^{2}}{64 \pi} M_{\tau^{\prime}}\left(1+2 r_{Z}\right)\left(1-r_{Z}\right)^{2},
\end{gathered}
$$

in both the singlet and doublet VLL models, while

$$
\begin{aligned}
\Gamma\left(\tau^{\prime} \rightarrow W \nu\right) \\
= \begin{cases}\frac{\epsilon^{2}}{32 \pi} M_{\tau^{\prime}}\left(1+2 r_{W}\right)\left(1-r_{W}\right)^{2}, & (\text { singlet VLL model }), \\
0, & \text { (doublet VLL model) },\end{cases}
\end{aligned}
$$

where $r_{X} \equiv M_{X}^{2} / M_{\tau^{\prime}}^{2}$ for each of $X=h, Z, W$. In the decays to $Z$ and $W$, the factors $\left(1+2 r_{Z}\right)$ and $\left(1+2 r_{W}\right)$ can be understood as coming from the longitudinal (1) and transverse $\left(2 r_{X}\right)$ components of the weak vector bosons. The longitudinal components can in turn be understood as essentially the Goldstone modes that are eaten by the vector bosons to obtain their masses. This illustrates the usual Goldstone equivalence principle, which implies that for the limit of large $M_{\tau^{\prime}}$ the branching ratios should approach

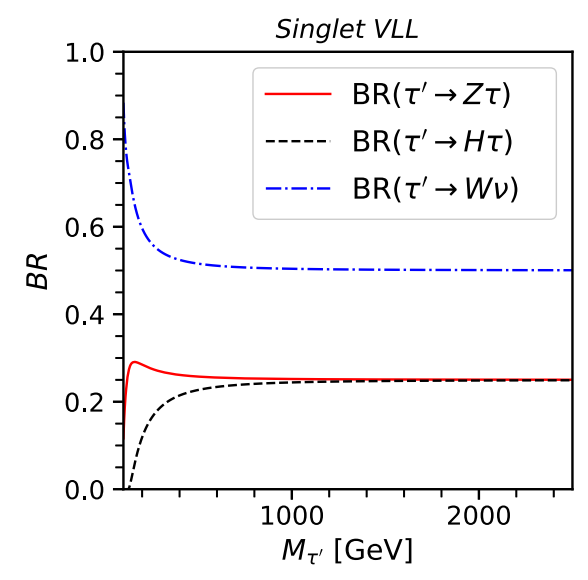

$$
\begin{aligned}
& {\left[\operatorname{BR}\left(\tau^{\prime} \rightarrow h \tau\right), \operatorname{BR}\left(\tau^{\prime} \rightarrow Z \tau\right), \operatorname{BR}\left(\tau^{\prime} \rightarrow W \nu\right)\right]} \\
& \quad= \begin{cases}{[0.25,0.25,0.5]} & (\text { singlet VLL model }), \\
{[0.5,0.5,0]} & (\text { doublet VLL model }) .\end{cases}
\end{aligned}
$$

In Fig. 4, we plot the branching ratios for the $\tau^{\prime}$ decays in the two models, as a function of the mass $M_{\tau^{\prime}}$, showing the asymptotic approach to the Goldstone equivalence limit.

The doublet VLL model therefore has the following final states:

$$
Z Z \tau^{-} \tau^{+}, \quad Z h \tau^{-} \tau^{+}, \quad h h \tau^{-} \tau^{+}
$$

from $\tau^{\prime}$ pair-production Eq. (2.6),

$$
W^{+} W^{-} \tau^{-} \tau^{+}
$$

from $\nu^{\prime}$ pair-production Eq. (2.7), and

$$
Z W^{ \pm} \tau^{-} \tau^{+}, \quad h W^{ \pm} \tau^{-} \tau^{+}
$$

from $\tau^{\prime} \nu^{\prime}$ production Eqs. (2.8) and (2.9). Besides the three signals in Eq. (2.15), the singlet VLL model also has final states

$Z W^{ \pm} \tau^{\mp}+E_{T}^{\mathrm{miss}}, \quad h W^{ \pm} \tau^{\mp}+E_{T}^{\mathrm{miss}}, \quad W^{+} W^{-}+E_{T}^{\text {miss }}$,

with the last being the largest in the minimal singlet VLL model.

With the above branching ratios for the minimal singlet VLL model, the analysis below does not find any reach for any of the signal regions at any of the collider options considered below, due to the low cross section and the unfortunate large branching ratio for $\tau^{\prime} \rightarrow W \nu$. Therefore, we instead consider a class of nonminimal models which have the feature that the lightest new fermion is still a charged, mostly isosinglet $\tau^{\prime}$, but with other new fermions

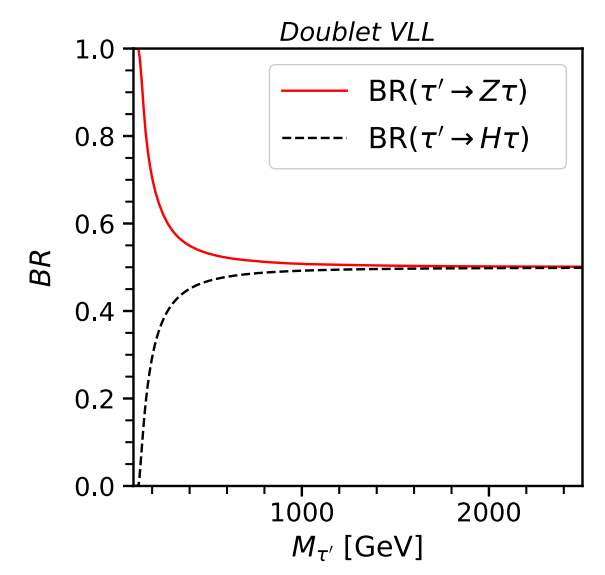

FIG. 4. The branching ratios for $\tau^{\prime} \rightarrow W \nu$ and $Z \tau$ and $h \tau$, as a function of $M_{\tau^{\prime}}$, for the singlet VLL model (left) and the doublet VLL model (right), showing the rapid approach to the Goldstone boson equivalence limit for larger masses. 
much heavier and therefore not contributing to the production. Thus, the new particle content first accessible to colliders is the same as in the minimal singlet VLL model, but the mixing with the much heavier vectorlike fermions allows the branching ratios of $\tau^{\prime}$ to be essentially arbitrary among the three final states listed above. We therefore present results for modified singlet VLL models, with the same $\tau^{\prime+} \tau^{\prime-}$ production cross section as the minimal singlet VLL model, but with branching ratios set to the following three subcases:

$\operatorname{BR}\left(\tau^{\prime} \rightarrow h \tau\right)=1, \quad$ "Higgs-philic singlet VLL,"

$$
\begin{gathered}
\operatorname{BR}\left(\tau^{\prime} \rightarrow Z \tau\right)=1, \quad \text { "Z-philic singlet VLL," } \\
\operatorname{BR}\left(\tau^{\prime} \rightarrow h \tau\right)=\operatorname{BR}\left(\tau^{\prime} \rightarrow Z \tau\right)=0.5, \\
\text { "W-phobic singlet VLL." }
\end{gathered}
$$

Realizations of these simplified models, from appropriate limits of mixing a vectorlike isosinglet fermion with a much heavier vectorlike isodoublet fermion, are discussed in the Appendix.

For signal simulation, we have input a Lagrangian which governs the production and decay of the doublet VLL and the singlet VLL models (as discussed above) into FEYNRULES v2.3 [83], a MATHEMATICA package, to obtain the Feynman rules as a Universal FEYNRULES Output (UFO) file, which is then imported into MADGRAPH5_AMC@NLO v2.6.5 [84]. ${ }^{4}$ We considered Standard Model production of the final states $W Z, Z Z$, $t \bar{t} Z, t \bar{t} W, h W, h Z, t \bar{t} h, W W W, W W Z, Z Z W$, and $Z Z Z$ as the main backgrounds contributing to multilepton final states. We did not include reducible backgrounds such as $W+$ jets, $Z+$ jets, and $W Z+$ jets, where one or more jets fake a tau. While they could be important, we do not have a way of reliably estimating them for unknown future detectors. Therefore it must be kept in mind that if they are large, our projections may be too optimistic.

Both signal and background events were generated using MADGRAPH at leading order (LO). The decay couplings in the (VLL) Lagrangian are left as free parameters in the model file of FEYNRULES, so that one has the flexibility to adjust them during the run time of MADGRAPH. The numerical values of the decay couplings that are used are actually not important (as long as they are not too small or too large), because in each separate MADGRAPH run the decays of VLL were forced into one of the individual channels mentioned above, and later the event samples were normalized using the branching ratios shown in Fig. 4. We calculated the cross sections for signal at LO and

\footnotetext{
${ }^{4}$ Electronic input files relevant for VLL models are available from the authors on request.
}

normalized the background cross sections as calculated at next-to-leading order, using MADGRAPH. While using MADGRAPH, we used their default set of parton distribution functions based on NNPDF2.3 set [85]. We used PYTHIA 8.2 $[86,87]$ for showering and hadronization. Then, DELPHES 3.4 [88] was used for detector simulation. We have used the default DELPHES tagging efficiencies and misidentification rates for taus and b-jets, where the tau tagging efficiency is 0.6 , and the tau misidentification rate for QCD jets is 0.01 . To increase the yield for background events to pass cuts described below, we forced every particle, except for the Standard Model (SM) Higgs boson $(h)$, to decay into leptons (including taus) and/or jets, such that they contribute to multilepton final states with at least three leptons. For generation of both signal and background events, we only considered the decay of $h$ into $W^{+} W^{-}, Z Z$, and $\tau^{+} \tau^{-}$, ignoring all other decays, and have normalized using the branching ratios of $h$ from HDECAY [89]. Thus each signal or background containing one or more $h$ is split into three signal or background components per $h$. We generated at least $10^{5}$ events for each signal component at each mass point, and at least $10^{6}$ events for each background component, at each of $\sqrt{s}=(14,27,70,100) \mathrm{TeV}$. These numbers of generated events are usually sufficient, with a few exceptions noted below. In the real world, better background determinations may come from data.

In our analysis, we first impose object cuts on leptons $\ell=e, \mu, \tau$, where, from now on, $\tau$ refers to a tau lepton that undergoes a hadronic decay. These include minimum $p_{T}$ cuts that depend on the collider option and are therefore listed in the subsections below. In all cases, the lepton candidates are required to pass the following further cuts on pseudorapidity $\eta$ and isolation from other lepton candidates or jets,

$$
|\eta|<2.5
$$

$$
\Delta R_{l, l^{\prime}}>0.1 \quad\left(\text { for each } \ell, \ell^{\prime}=e, \mu, \tau\right)
$$

$$
\left.\Delta R_{l, j}>0.3 \quad \text { (for each jet and } \ell=e, \mu, \tau\right) .
$$

Here, $\Delta R=\sqrt{(\Delta \phi)^{2}+(\Delta \eta)^{2}}$ as is usual.

Events are then selected with at least three leptons, of which at least two must be $e$ or $\mu$. The leading $e$ or $\mu$ lepton is also required to satisfy a minimum $p_{T}$ trigger requirement that depends on the collider option and is therefore listed separately in each of the subsections below. We also veto $b$-jets to reduce large backgrounds from $t \bar{t}$ production processes. Thus our event preselection common to all signal regions is

$$
\begin{gathered}
N(e, \mu, \tau) \geq 3, \\
N(e, \mu) \geq 2,
\end{gathered}
$$




$$
\begin{gathered}
p_{T}^{e_{1}} \text { or } p_{T}^{\mu_{1}}>p_{T}^{\text {trigger }}, \\
N(b \text {-jets })=0 .
\end{gathered}
$$

Events with no $e^{+} e^{-}$or $\mu^{+} \mu^{-}$pair with invariant mass within $15 \mathrm{GeV}$ of $M_{Z}$ are referred to below as "no $Z$," and events with exactly two $e / \mu$ that have same-sign charges will be labeled as "SS." We then considered the following six distinct types of signal regions:

$$
2 \mathrm{SS} e / \mu+\geq 1 \tau
$$

$$
\begin{gathered}
2 \mathrm{SS} e / \mu+\geq 1 \tau \quad \text { with } \quad E_{T}^{\text {miss }}>150 \mathrm{GeV}, \\
\geq 3 e / \mu+1 \tau \\
\geq 3 e / \mu+1 \tau, \text { no } Z \\
\geq 2 e / \mu+2 \tau \\
\geq 2 e / \mu+2 \tau, \text { no } Z .
\end{gathered}
$$
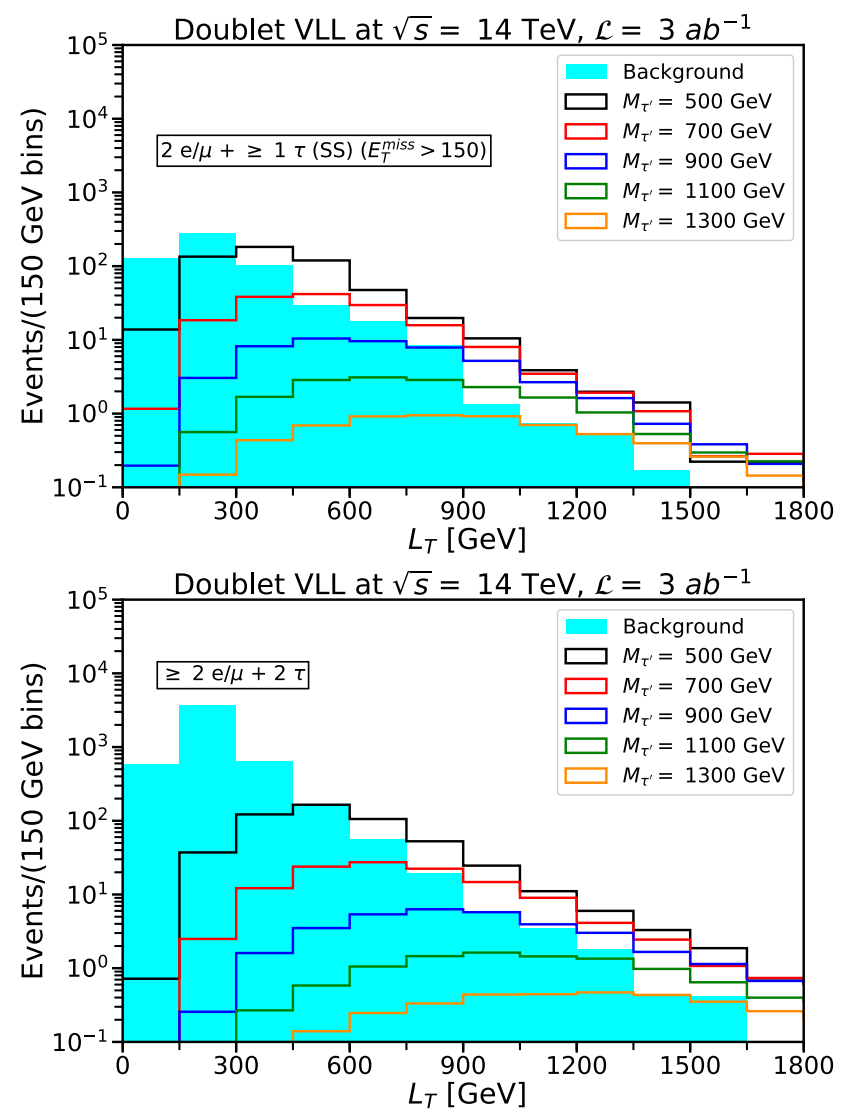

Finally, in each of these signal regions, we imposed a minimum lower bound cut on $L_{T}$, which is defined to be the sum of the transverse momentum of all leptons

$$
L_{T}=\sum_{\ell=e, \mu, \tau} p_{T}(\ell)
$$

We varied the choice of this cut to obtain good exclusion and discovery reach simultaneously for each signal region and collider option and assumed fractional background uncertainty $\Delta_{b} / b=(0.1,0.2,0.5)$. However, we have only done a very rough optimization for the $L_{T}$ cut, in part because the optimization is different for exclusion and for discovery, and also because the Monte Carlo simulations are only an approximation of the actual experimental capabilities, which will rely on detector designs yet to be determined. In general, the results found below reflect that the choice of cut on $L_{T}$ increases with the mass $M_{\tau^{\prime}}$ at the edge of the reach. Also, for convenience, we always choose the same $L_{T}$ cut for both exclusion and discovery, even though an optimized cut would likely be somewhat different for the two cases.
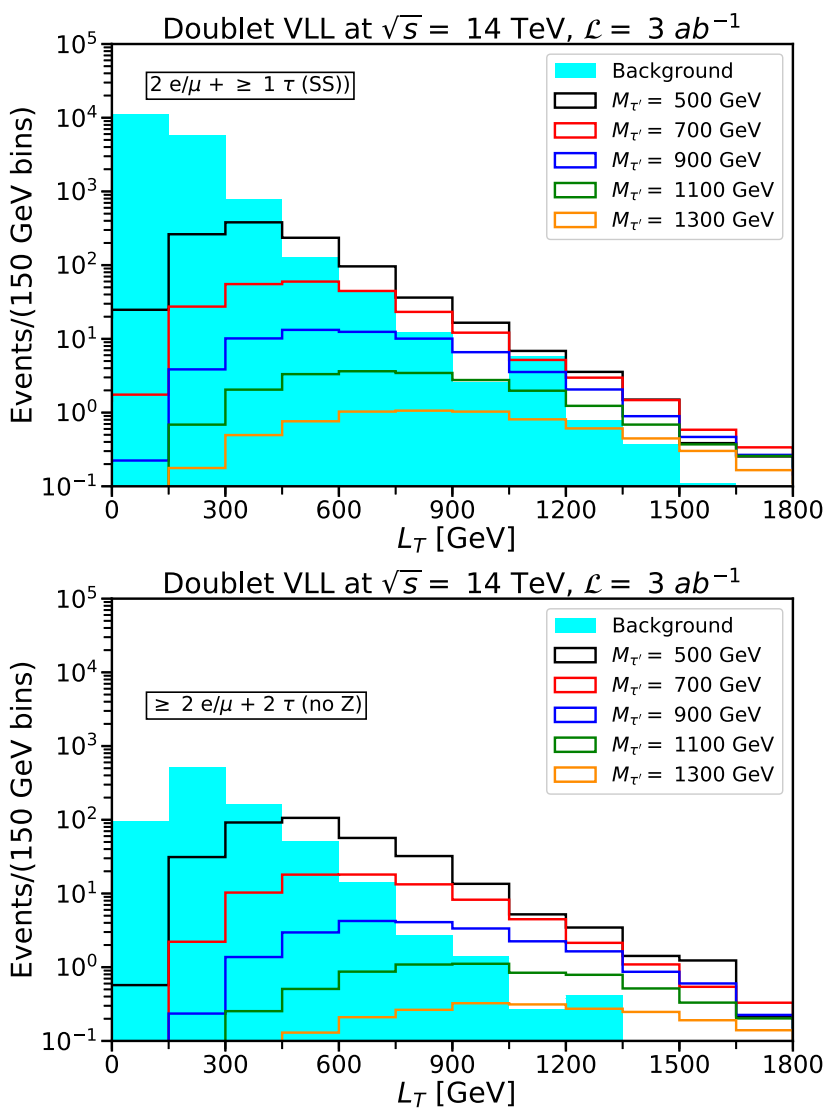

FIG. 5. $L_{T}$ event distributions for total background (shaded) and doublet VLL models (lines) for $p p$ collisions at $\sqrt{s}=14$ TeV with an integrated luminosity $\mathcal{L}=3 \mathrm{ab}^{-1}$. Five different masses $M_{\tau^{\prime}}=M_{\nu^{\prime}}=500,700,900,1100$, and $1300 \mathrm{GeV}$ are shown in each panel. The four panels show results for the four best signal regions, as labeled. 
Note that the signal regions considered in Eqs. (2.29)(2.34) are far from exclusive of each other. Therefore, to be conservative we have not attempted to combine them, although doing so could lead to some extension of the reach prospects.

\section{RESULTS FOR THE HL-LHC COLLIDER}

In this section, we discuss the possibility of exclusion or discovery of both doublet and singlet VLL (including the minimal and nonminimal versions) at $\sqrt{s}=14 \mathrm{TeV}$ with $3 \mathrm{ab}^{-1}$ of $p p$ collisions. In addition to the pseudorapidity, isolation, and other requirements of Eqs. (2.22)-(2.28), we require all leptons including hadronic tau candidates to satisfy

$$
p_{T}^{\ell}>15 \mathrm{GeV} \text {. }
$$

Additionally, the leading $e$ or $\mu$ in each event is required to satisfy a trigger requirement

$$
p_{T}^{e_{1}} \text { or } p_{T}^{\mu_{1}}>30 \mathrm{GeV}
$$
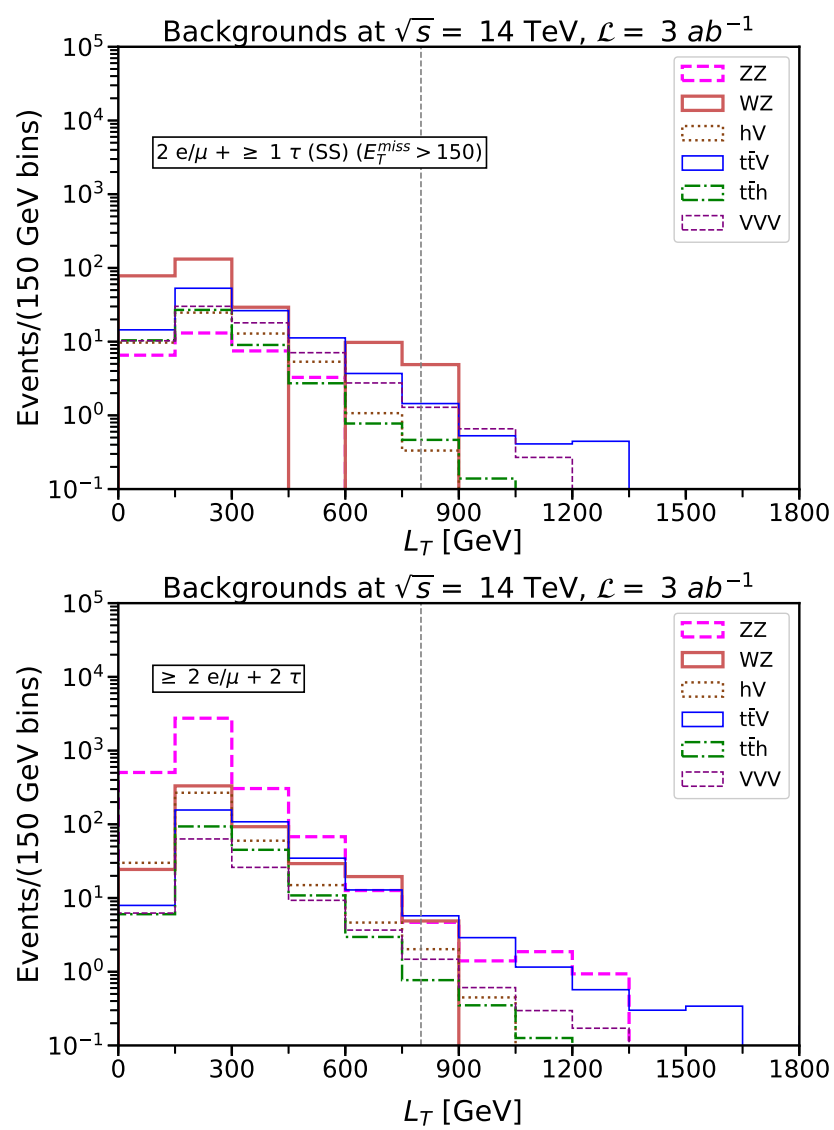

We then considered the six signal regions mentioned in Eqs. (2.29)-(2.34).

\section{A. Doublet VLL model}

In Fig. 5, we show the $L_{T}$ distributions for the best four of these signal regions, for five different choices of $M_{\tau^{\prime}}$ as labeled, and for the total of all backgrounds shown as the shaded histogram. For $p p$ collisions with $\sqrt{s}=14 \mathrm{TeV}$ with $3 \mathrm{ab}^{-1}$, we found that the expected reach is approximately maximized if we then choose a cut $L_{T}>800 \mathrm{GeV}$.

Figure 6 shows the $L_{T}$ distributions for all background components, for the four best signal regions as labeled. The $L_{T}$ cut is shown in the figure as a vertical dashed line. After imposing the $L_{T}$ cut, the dominant SM backgrounds are $W Z, t \bar{t} V$, and $V V V$ (where $V=W, Z$ ) in the two signal regions with $2 \mathrm{SS} e / \mu+\geq 1 \tau$, while the dominant SM backgrounds are $t \bar{t} V$ and $Z Z$ in the signal region with $\geq 2 e / \mu+2 \tau$, and $t \bar{t} V$ and $t \bar{t} h$ in the signal region with $\geq 2 e / \mu+2 \tau$ (no $Z$ ).

Figure 7 shows the resulting median expected significances for exclusion (left panels) and discovery (right panels), for $\Delta_{b} / b=0.1$ (top row), 0.2 (middle row), and 0.5 (bottom row), with the cut requirement $L_{T}>800 \mathrm{GeV}$
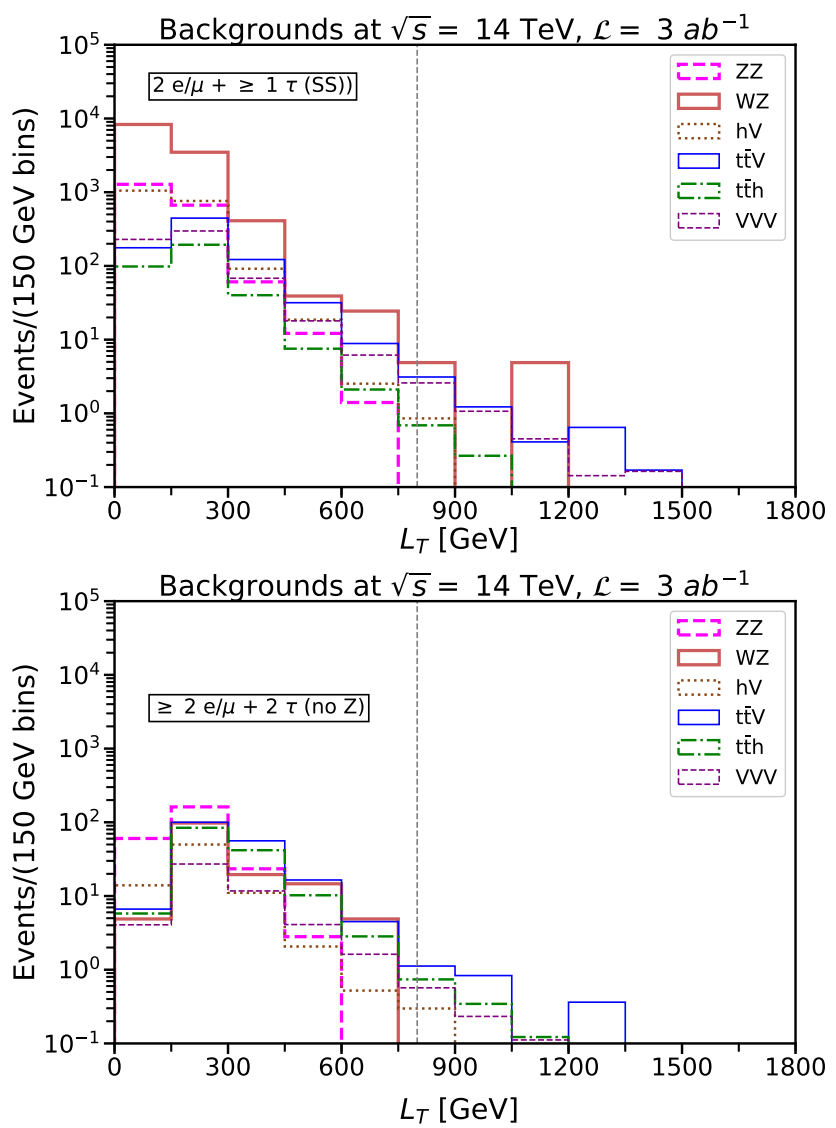

FIG. 6. $L_{T}$ event distributions for all processes contributing to total SM background, for $p p$ collisions at $\sqrt{s}=14 \mathrm{TeV}$ with an integrated luminosity $\mathcal{L}=3 \mathrm{ab}^{-1}$. The four panels show results for the four best signal regions, as labeled. The vertical dashed line in all four panels shows our choice of $L_{T}$ cut. 

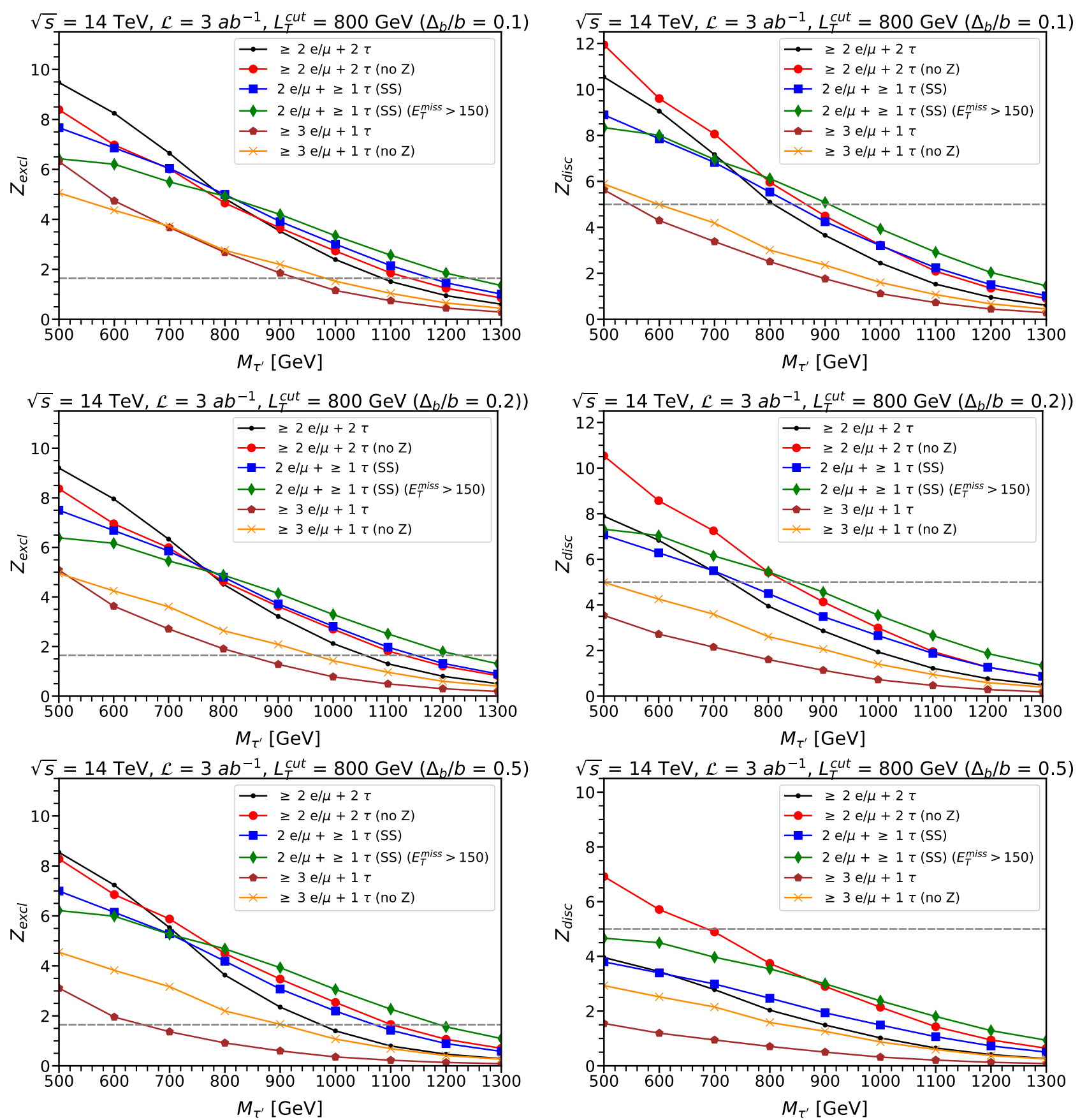

FIG. 7. The median expected significances for exclusion $Z_{\text {excl }}$ (left) and discovery $Z_{\text {disc }}$ (right) as a function of $M_{\tau^{\prime}}$ in the doublet VLL model, for $p p$ collisions at $\sqrt{s}=14 \mathrm{TeV}$ with integrated luminosity $\mathcal{L}=3 \mathrm{ab}^{-1}$, for six different signal regions as described in the text, each including a cut $L_{T}>800 \mathrm{GeV}$. The fractional uncertainty in the background is assumed to be $\Delta_{b} / b=0.1$ (top), 0.2 (middle), and 0.5 (bottom).

imposed. In all cases, the best signal regions for both exclusion and discovery scenarios are the ones with 2 SS $e / \mu+\geq 1 \tau$, with the additional requirement $E_{T}^{\text {miss }}>$ $150 \mathrm{GeV}$ providing slightly more reach. The two signal regions with $\geq 2 e / \mu+2 \tau$ give slightly less reach at higher masses, but could actually provide comparable exclusion significances for lower masses.
From Fig. 7 we conclude that a $14 \mathrm{TeV}$ high-luminosity LHC with $3 \mathrm{ab}^{-1}$ should be able to exclude doublet VLLs with $M_{\tau^{\prime}}$ up to about $1250 \mathrm{GeV}$ if they are indeed absent or discover them if the mass is less than about $900 \mathrm{GeV}$, assuming that the future background determination from data is subject to uncertainties of order $10 \%$ or less. The figure also shows that prospects for exclusion are much less 


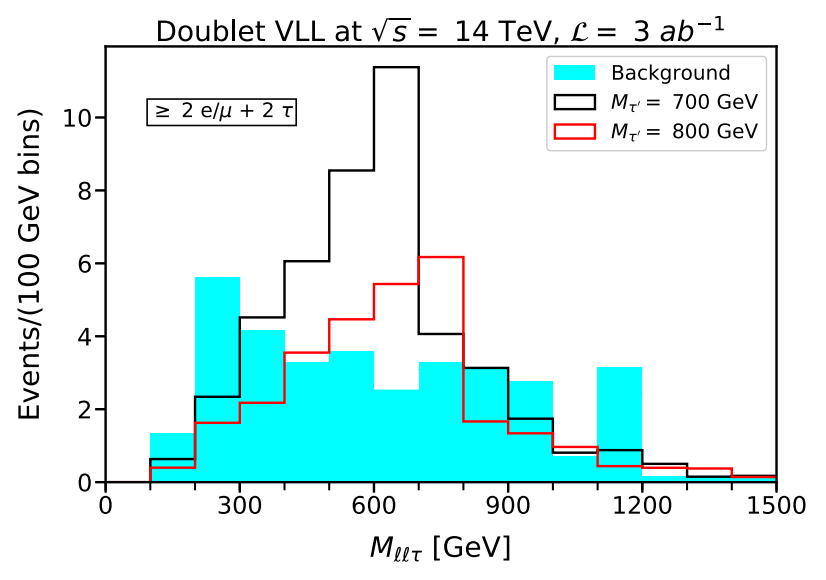

FIG. 8. The event distributions for three-body invariant mass of $\tau^{ \pm} e^{+} e^{-}$or $\tau^{ \pm} \mu^{+} \mu^{-}$, for total background (shaded) and doublet VLLs (lines), such that the $e^{+} e^{-}$or $\mu^{+} \mu^{-}$pair have an invariant mass within $10 \mathrm{GeV}$ of $M_{Z}$ in a signal region with $\geq 2 e / \mu+2 \tau$, with the cut $L_{T}>800 \mathrm{GeV}$ imposed. Two different masses $M_{\tau^{\prime}}=M_{\nu^{\prime}}=700$ and $800 \mathrm{GeV}$ are shown.
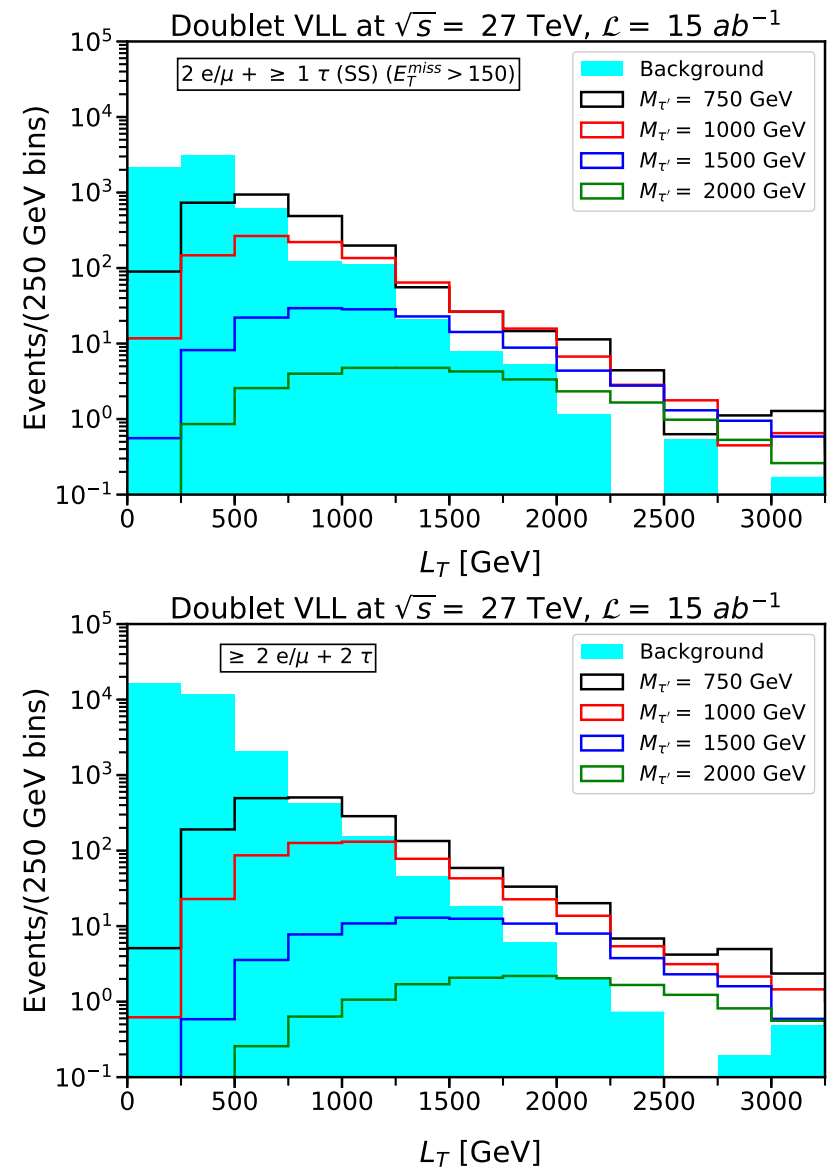

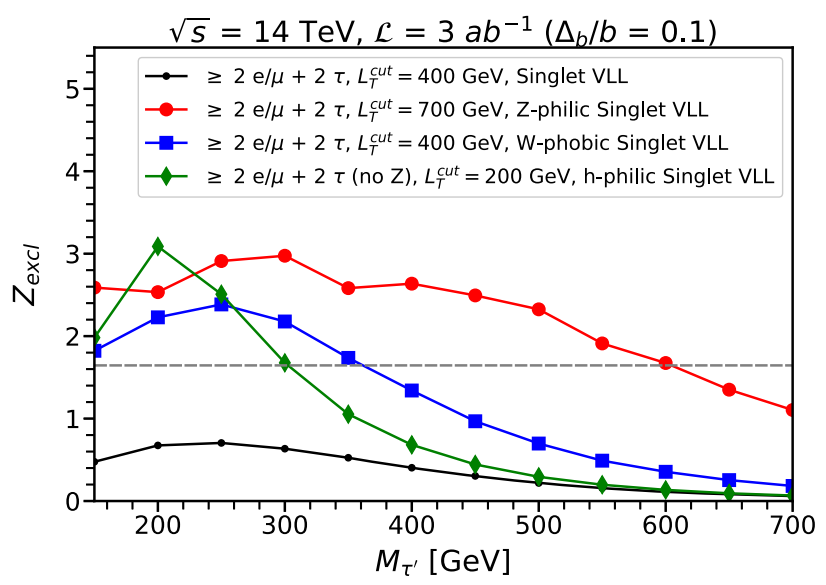

FIG. 9. The median expected significances for exclusion $Z_{\text {excl }}$ as a function of $M_{\tau^{\prime}}$ in the singlet VLL models, for $p p$ collisions at $\sqrt{s}=14 \mathrm{TeV}$ with integrated luminosity $\mathcal{L}=3 \mathrm{ab}^{-1}$, for the best signal region for each of the singlet VLL models, including a cut on $L_{T}$ as shown in the plot. The fractional uncertainty in the background is assumed to be $\Delta_{b} / b=0.1$.
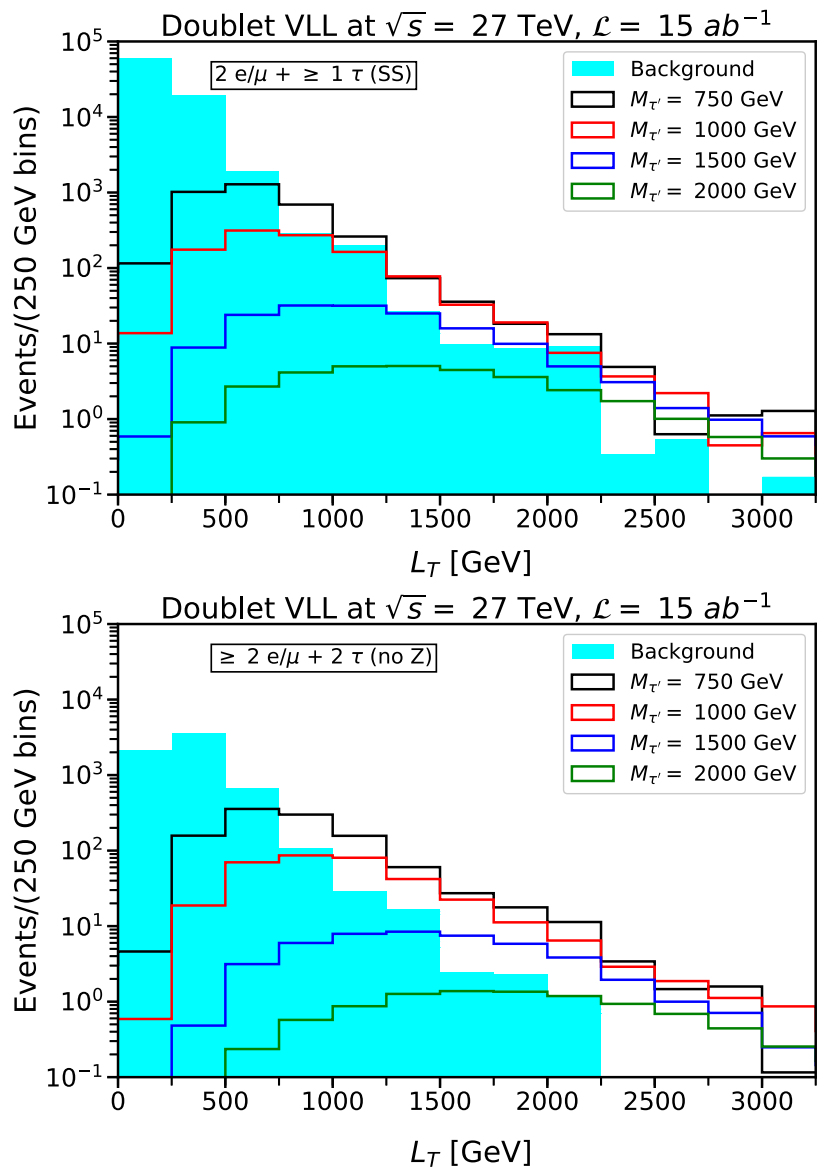

FIG. 10. $L_{T}$ event distributions for total background (shaded) and doublet VLL models (lines), for $p p$ collisions at $\sqrt{s}=27$ TeV with an integrated luminosity $\mathcal{L}=15 \mathrm{ab}^{-1}$. Four different masses $M_{\tau^{\prime}}=M_{\nu^{\prime}}=750,1000,1500$, and $2000 \mathrm{GeV}$ are shown in each panel. The four panels show results for the four best signal regions, as labeled. 
sensitive to uncertainty in the background than the prospects for discovery. For $\Delta_{b} / b=0.5$, one can still expect to exclude doublet VLLs up to about $M_{\tau^{\prime}}=1190 \mathrm{GeV}$ or discover them if the mass is less than about $690 \mathrm{GeV}$, but the latter has already been excluded at $95 \%$ confidence level by CMS [70,71].

In the case that there are enough events for a clear discovery, one can also hope to measure the mass of the $\tau^{\prime}$. In Fig. 8, we show the event distributions for the threebody invariant mass of $\tau^{ \pm} e^{+} e^{-}$or $\tau^{ \pm} \mu^{+} \mu^{-}$, for the signal region with $\geq 2 e / \mu+2 \tau$, for two different choices of input $M_{\tau^{\prime}}$, and for the total of all backgrounds shown as the shaded histogram. (Combinatorial backgrounds from wrong associations of the lepton pair and tau in the signal sample are of course also present and included.) Here, we require the two-body invariant mass of the $e^{+} e^{-}$or $\mu^{+} \mu^{-}$ pair to be within $10 \mathrm{GeV}$ of $M_{Z}$. Additionally, we also impose the cut $L_{T}>800 \mathrm{GeV}$. From Fig. 8, we observe that the distributions for doublet VLLs are peaked just below their respective masses, which gives a possibility to measure the masses of doublet VLLs, if they are indeed discovered. At $\sqrt{s}=14 \mathrm{TeV}$, the range of masses that one can hope to measure in this way is limited by the present exclusion up to $M_{\tau^{\prime}}=790 \mathrm{GeV}$ by CMS [71] and by the fact that the cross section decreases rapidly for higher masses.

\section{B. Singlet VLL models}

Singlet VLLs are much more challenging than doublet VLL, due to their much smaller production cross sections. For the minimal singlet model, we find no possible exclusion or discovery at $\sqrt{s}=14 \mathrm{TeV}$ with integrated luminosity of $3 \mathrm{ab}^{-1}$. And, for the nonminimal singlet models, we find some exclusion possibility, but with no prospects for discovery. In view of the difficulties involved, below we consider only the case where the uncertainty in the background is $\Delta_{b} / b=0.1$.

To maximize the exclusion reach in the nonminimal cases, we chose a cut $L_{T}>700 \mathrm{GeV}$ for the $Z$-philic singlet VLL, $L_{T}>400 \mathrm{GeV}$ for the $W$-phobic singlet VLL, and $L_{T}>200 \mathrm{GeV}$ for the Higgs-philic singlet VLL. Figure 9 shows the resulting median expected significances for exclusion, for $\Delta_{b} / b=0.1$, for the best signal region for each of the singlet VLL models, with the cuts on $L_{T}$ imposed. In all the models, the best signal region
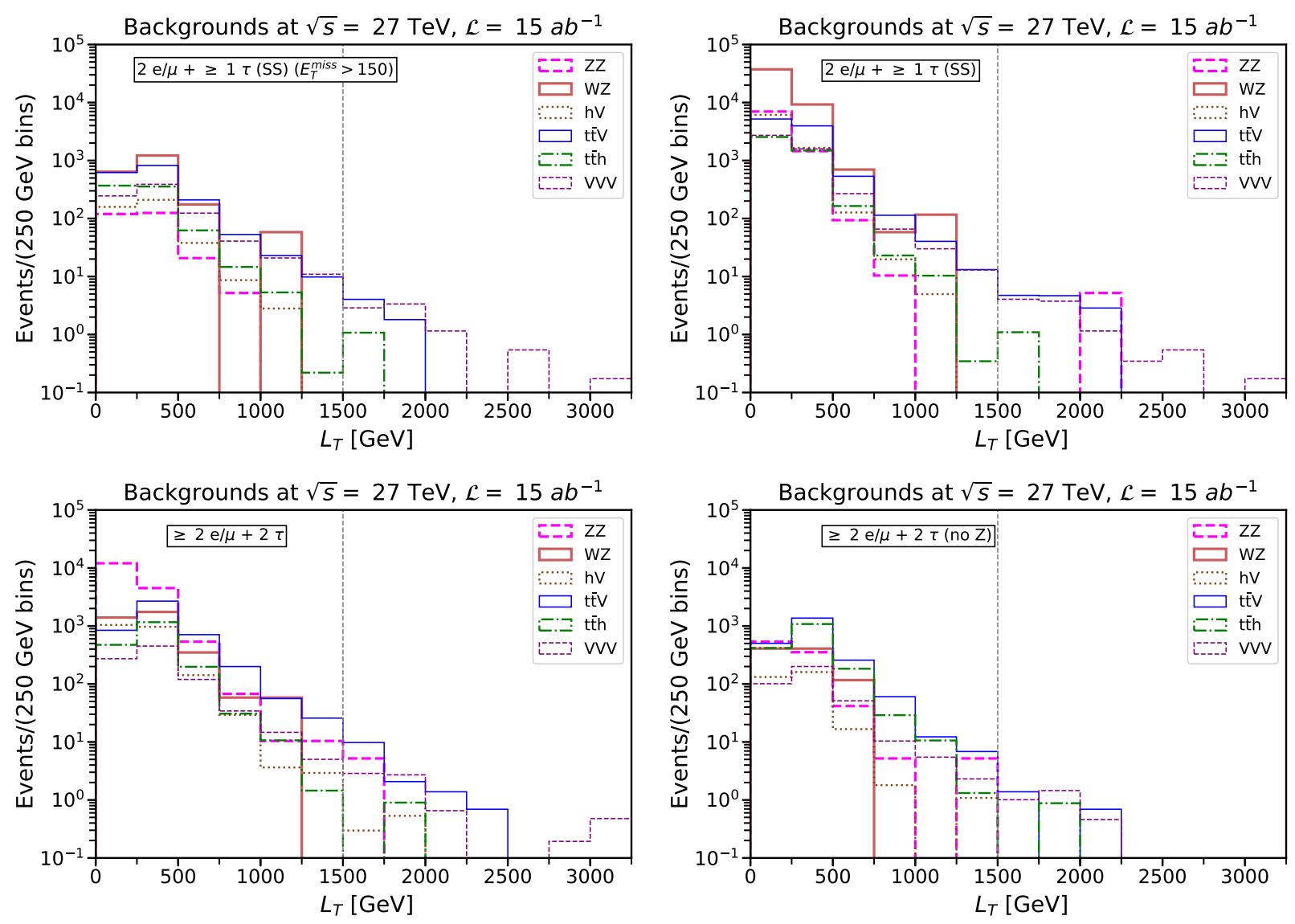

FIG. 11. $L_{T}$ event distributions for all processes contributing to total SM background, for $p p$ collisions at $\sqrt{s}=27 \mathrm{TeV}$ with an integrated luminosity $\mathcal{L}=15 \mathrm{ab}^{-1}$. The four panels show results for the four best signal regions, as labeled. The vertical dashed line in all four panels shows our choice of $L_{T}$ cut of $1500 \mathrm{GeV}$. 
for exclusion is the one which requires $\geq 2 e / \mu+2 \tau$. However, for the Higgs-philic singlet VLL model, the additional requirement of no $Z$ proved to be beneficial.

From Fig. 9, we conclude that assuming $\Delta_{b} / b=0.1$, a $14 \mathrm{TeV} p p$ collider with $3 \mathrm{ab}^{-1}$ should be able to exclude singlet VLLs with masses up to about $600 \mathrm{GeV}$ if they are
$Z$-philic, or exclude masses up to about $360 \mathrm{GeV}$ if they are $W$-phobic, or exclude masses up to about $300 \mathrm{GeV}$ if they are Higgs-philic. We find that there is no possible exclusion of singlet VLLs in the minimal model. Furthermore, there are no discovery prospects in minimal or nonminimal singlet VLL models.
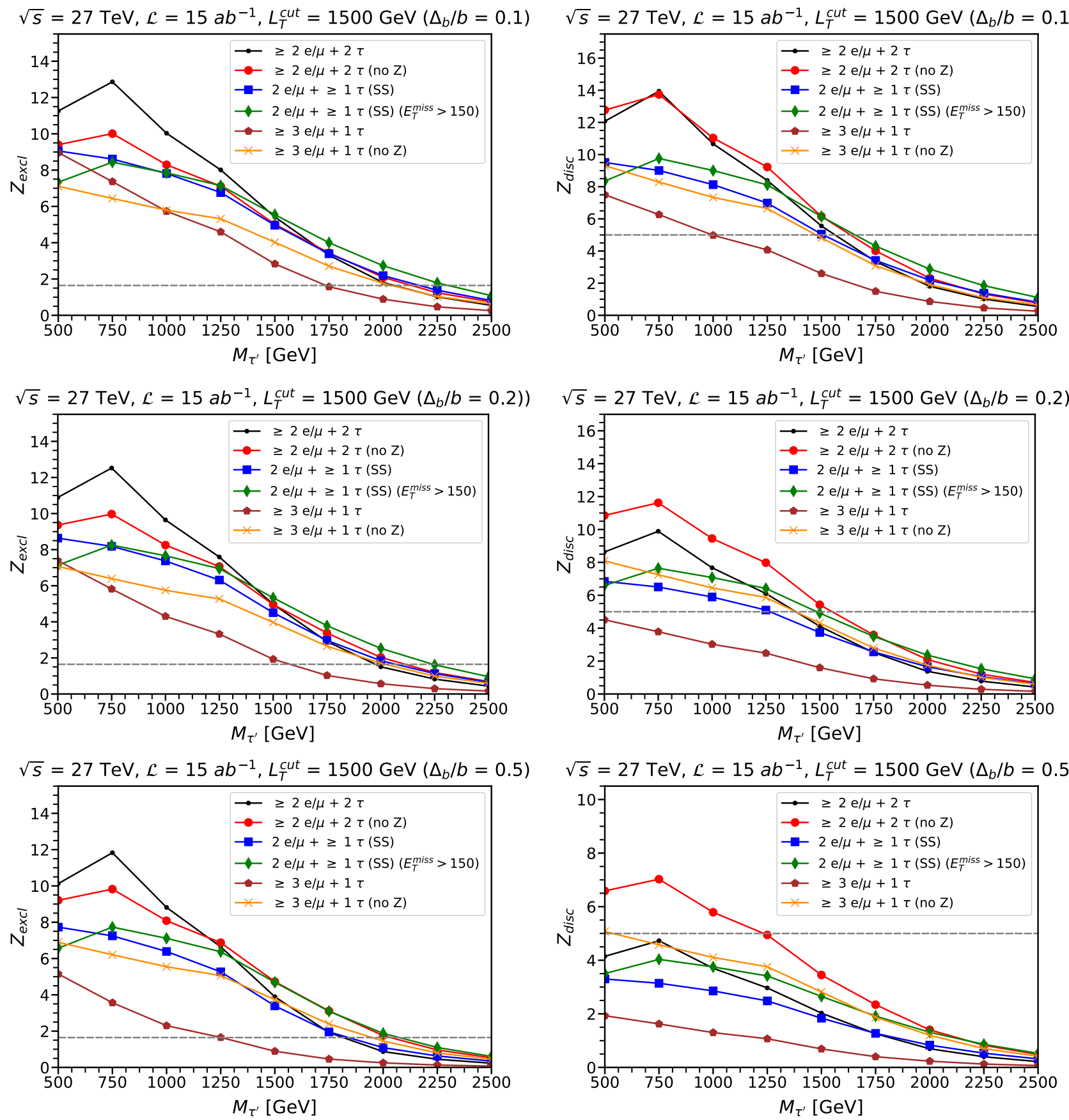

FIG. 12. The median expected significances for exclusion $Z_{\text {excl }}$ (left) and discovery $Z_{\text {disc }}$ (right) as a function of $M_{\tau^{\prime}}$ in the doublet VLL model, for $p p$ collisions at $\sqrt{s}=27 \mathrm{TeV}$ with integrated luminosity $\mathcal{L}=15 \mathrm{ab}^{-1}$, for six different signal regions as described in the text, each including a cut $L_{T}>1500 \mathrm{GeV}$. The fractional uncertainty in the background is assumed to be $\Delta_{b} / b=0.1$ (top), 0.2 (middle), and 0.5 (bottom). 


\section{RESULTS FOR THE HE-LHC COLLIDER}

In this section, we discuss the prospects for exclusion and discovery of VLLs at $\sqrt{s}=27 \mathrm{TeV}$ with $15 \mathrm{ab}^{-1}$ of $p p$ collisions in the six signal regions mentioned in Eqs. (2.29)-(2.34). All leptons including hadronic tau candidates are required to satisfy

$$
p_{T}^{\ell}>25 \mathrm{GeV},
$$

along with the same pseudorapidity, isolation, and other requirements of Eqs. (2.22)-(2.28), with a trigger requirement of

$$
p_{T}^{e_{1}} \text { or } p_{T}^{\mu_{1}}>50 \mathrm{GeV}
$$

\section{A. Doublet VLL model}

In Fig. 10, we show the $L_{T}$ distributions for the best four of these signal regions, for four different choices of $M_{\tau^{\prime}}$ as labeled, and for the total of all backgrounds shown as the shaded histogram. The cut chosen to enhance the reach for exclusion and discovery in this case was $L_{T}>1500 \mathrm{GeV}$.

Figure 11 shows the $L_{T}$ distributions for all background components, for the four best signal regions as labeled. The $L_{T}$ cut is shown in the figure as a vertical dashed line. After imposing the $L_{T}$ cut, the most important SM backgrounds are $t \bar{t} V$ and $V V V$ in all four of these signal regions.

Figure 12 shows the $Z_{\text {excl }}$ (left panels) and $Z_{\text {disc }}$ (right panels) as a function of $M_{\tau^{\prime}}$, for $\Delta_{b} / b=0.1$ (top row), 0.2 (middle row), and 0.5 (bottom row), with the cut $L_{T}>1500 \mathrm{GeV}$ imposed. The signal region with $2 \mathrm{SS}$ $e / \mu+\geq 1 \tau$ with $E_{T}^{\text {miss }}>150 \mathrm{GeV}$ has the farthest mass reach. However, at lower masses the two signal regions with $\geq 2 e / \mu+2 \tau$ give slightly better exclusion and discovery significances.

By looking at Fig. 12, we conclude that a $27 \mathrm{TeV}$ highenergy LHC with $15 a^{-1}$ could exclude doublet VLLs with masses up to about $2300 \mathrm{GeV}$ or discover them if the mass is less than about $1700 \mathrm{GeV}$, assuming the fractional uncertainty in the background to be 0.1 . On the other hand, if we assume $\Delta_{b} / b=0.5$, we would still be able to exclude doublet VLLs up to $M_{\tau^{\prime}}=2050 \mathrm{GeV}$ or discover them if the mass is less than about $1250 \mathrm{GeV}$. Just as in the case of the HL-LHC, from Fig. 12, a larger uncertainty in the background has a moderate effect on the prospects for exclusion, but a much larger impact on the prospects for discovery.

We again consider the prospects for observing a mass peak in the case where there are enough events for a clear discovery. In Fig. 13, we show the event distributions for the three-body invariant mass of $\tau^{ \pm} e^{+} e^{-}$or $\tau^{ \pm} \mu^{+} \mu^{-}$, for the signal region with $\geq 2 e / \mu+2 \tau$, for three different choices of $M_{\tau^{\prime}}$. The total of all backgrounds is shown as the shaded histogram. We require the two-body invariant mass of the

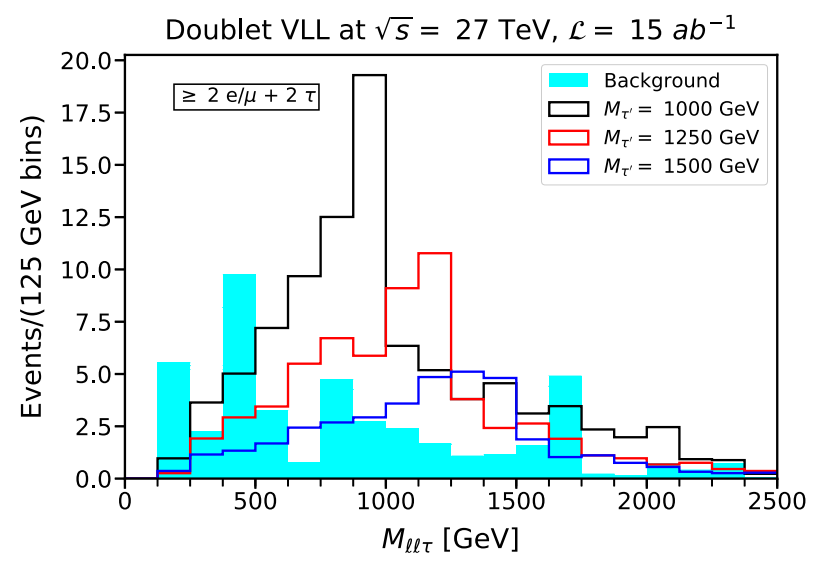

FIG. 13. The event distributions for three-body invariant mass of $\tau^{ \pm} e^{+} e^{-}$or $\tau^{ \pm} \mu^{+} \mu^{-}$, for total background (shaded) and doublet VLLs (lines), such that the $e^{+} e^{-}$or $\mu^{+} \mu^{-}$pair have an invariant mass within $10 \mathrm{GeV}$ of $M_{Z}$ in a signal region with $\geq 2 e / \mu+2 \tau$, with the cut $L_{T}>1500 \mathrm{GeV}$ imposed. Three different masses $M_{\tau^{\prime}}=M_{\nu^{\prime}}=1000,1250$, and $1500 \mathrm{GeV}$ are shown in the plot.

$e^{+} e^{-}$or $\mu^{+} \mu^{-}$pair to be within $10 \mathrm{GeV}$ of $M_{Z}$. Additionally, we also impose the cut $L_{T}>1500 \mathrm{GeV}$. From Fig. 13, we observe that the distributions for doublet VLLs are peaked just below their respective masses, which gives a possibility to measure the masses of doublet VLLs, if they are indeed discovered. For higher masses, statistical limitations and the combinatorial background mean that the mass determinations will be problematic.

\section{B. Singlet VLL models}

At $\sqrt{s}=27 \mathrm{TeV}$ with integrated luminosity of $15 \mathrm{ab}^{-1}$, we find that there is no exclusion or discovery reach for the minimal and the Higgs-philic singlet VLL models. For the other two nonminimal singlet models, we find some exclusion possibility, but again with no prospects for discovery.

We chose a cut $L_{T}>1400 \mathrm{GeV}$ for the $Z$-philic singlet VLL and $L_{T}>800 \mathrm{GeV}$ for the $W$-phobic singlet VLL, to maximize the exclusion reach. A cut $L_{T}>600 \mathrm{GeV}$ for the Higgs-philic singlet VLL and $L_{T}>800 \mathrm{GeV}$ for the minimal singlet VLL model were chosen for the best possible exclusion significance. Figure 14 shows the resulting median expected significances for exclusion, for $\Delta_{b} / b=0.1$, for the best signal region for each of the singlet VLL models, with the cuts on $L_{T}$ imposed, as mentioned above. The best signal region for exclusion, for all the singlet VLL models is the one which requires $\geq 2 e / \mu+2 \tau$, except that an additional requirement of no $Z$ gave better results for the Higgs-philic model.

From Fig. 14, we can conclude that a $27 \mathrm{TeV} p p$ collider with $15 \mathrm{ab}^{-1}$ could possibly exclude singlet VLLs with masses up to about $1200 \mathrm{GeV}$ in the Z-philic model, or exclude masses up to about $670 \mathrm{GeV}$ in the $W$-phobic model, but with no prospects for discovery. These results 


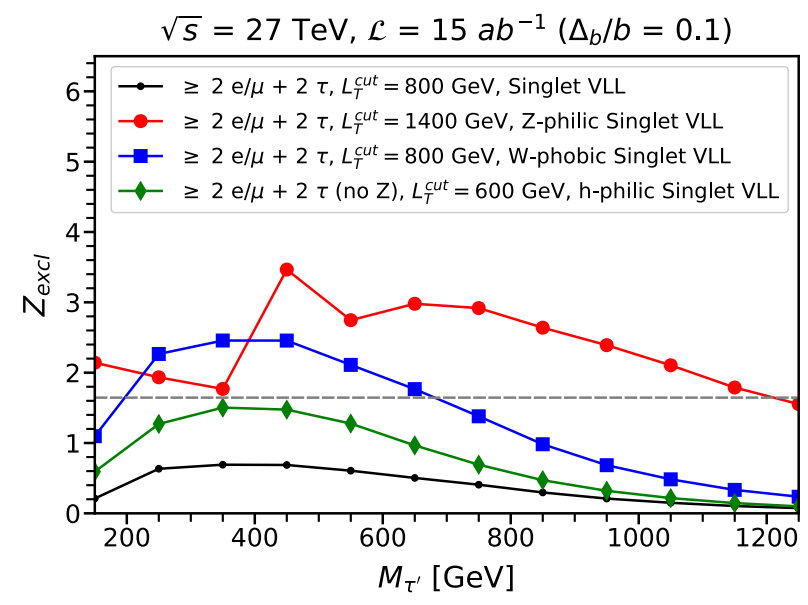

FIG. 14. The median expected significances for exclusion $Z_{\text {excl }}$ as a function of $M_{\tau^{\prime}}$ in the singlet VLL models, for $p p$ collisions at $\sqrt{s}=27 \mathrm{TeV}$ with integrated luminosity $\mathcal{L}=15 \mathrm{ab}^{-1}$, for the best signal region for each of the singlet VLL models, including a cut on $L_{T}$ as shown in the plot. The fractional uncertainty in the background is assumed to be $\Delta_{b} / b=0.1$.

assume a fractional uncertainty in the background of $\Delta_{b} / b=0.1$. In both minimal and the Higgs-philic singlet VLL models, there is no possibility for exclusion or
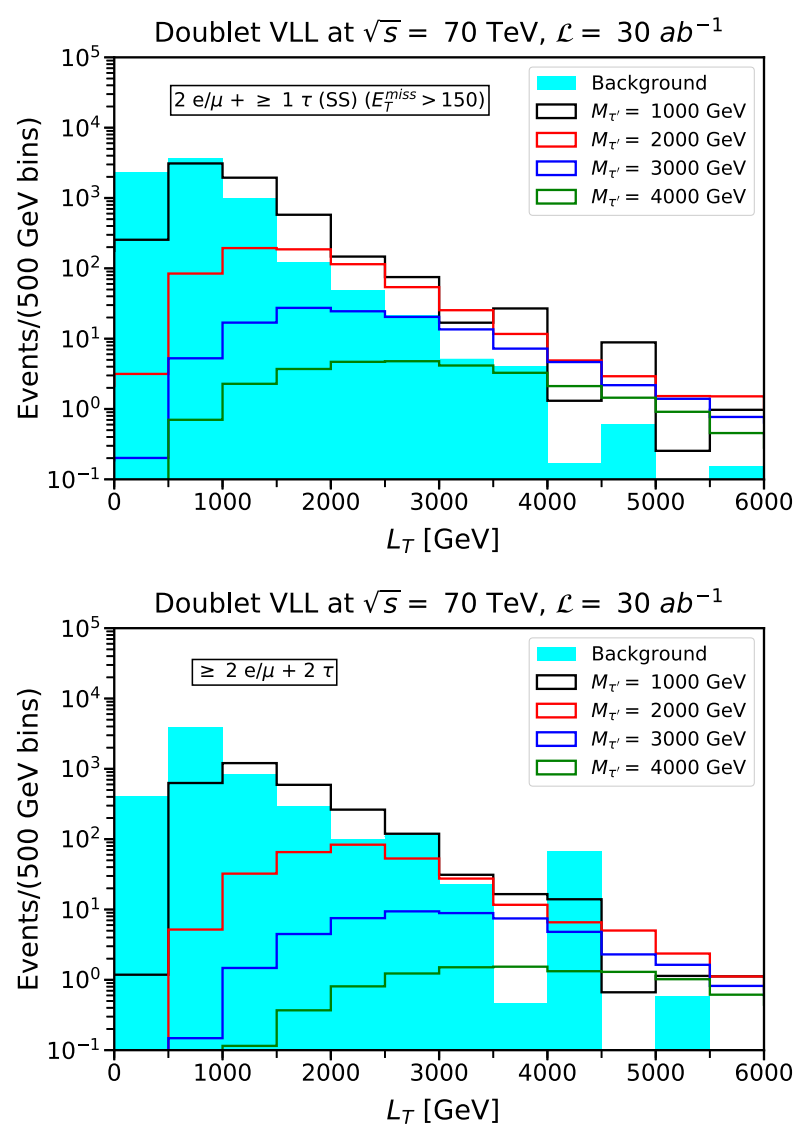

discovery. From the results for singlet VLL analyses for 14 and $27 \mathrm{TeV}$ colliders, we can note that the exclusion reach scaled approximately linearly with $\sqrt{s}$, for both $Z$ philic and the $W$-phobic singlet VLL models.

\section{RESULTS FOR A $p p$ COLLIDER WITH $\sqrt{s}=70 \mathrm{TeV}$}

In this section, we turn our attention to prospects for exclusion and discovery of VLLs at a possible future $p p$ collider at $\sqrt{s}=70 \mathrm{TeV}$, with integrated luminosity of $30 \mathrm{ab}^{-1}$ in the six signal regions mentioned in Eqs. (2.29)-(2.34). All the leptons including hadronic tau candidates are required to satisfy

$$
p_{T}^{\ell}>75 \mathrm{GeV}
$$

along with the same pseudorapidity, isolation, and other requirements of Eqs. (2.22)-(2.28), with at least one $e$ or $\mu$ satisfying a trigger requirement

$$
p_{T}^{e_{1}} \text { or } p_{T}^{\mu_{1}}>150 \mathrm{GeV} \text {. }
$$
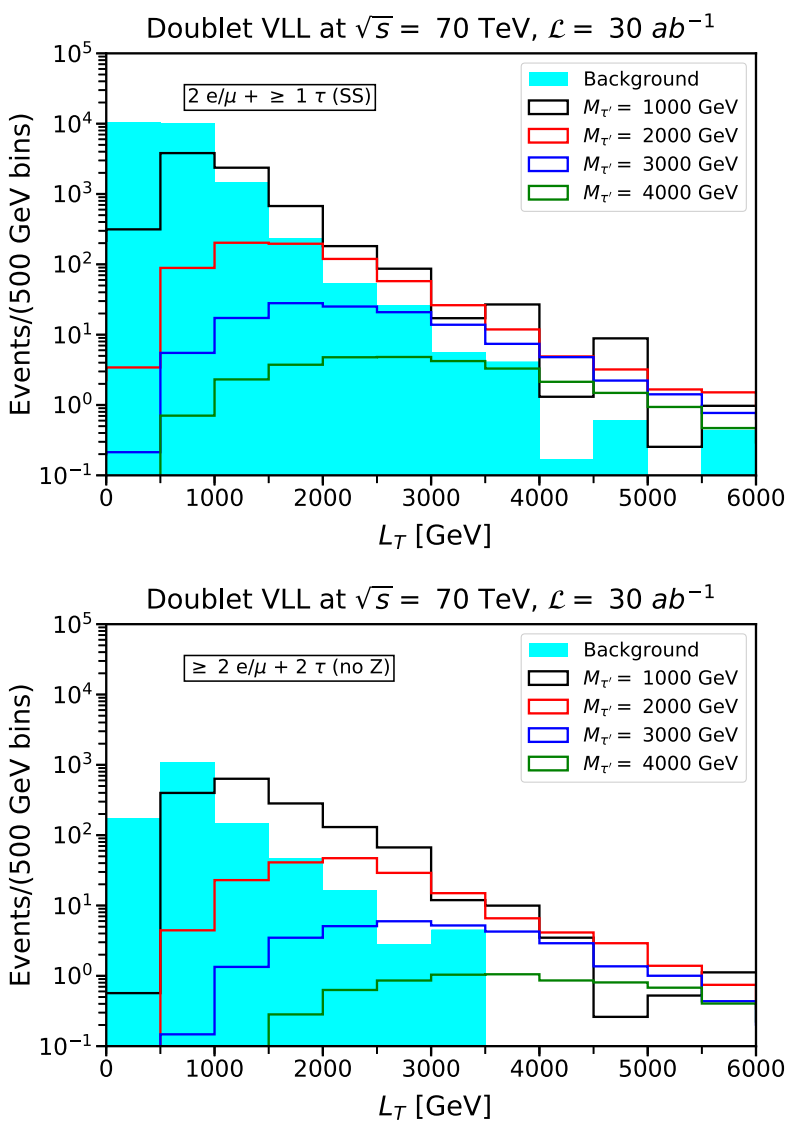

FIG. 15. $L_{T}$ event distributions for total background (shaded) and doublet VLL models (lines), for $p p$ collisions at $\sqrt{s}=70$ TeV with an integrated luminosity $\mathcal{L}=30 \mathrm{ab}^{-1}$. Four different masses $M_{\tau^{\prime}}=M_{\nu^{\prime}}=1000,2000,3000$, and $4000 \mathrm{GeV}$ are shown in each panel. The four panels show results for the four best signal regions, as labeled. 


\section{A. Doublet VLL model}

In Fig. 15, we show the $L_{T}$ distributions for the best four of the signal regions, for four different choices of $M_{\tau^{\prime}}$ as labeled, and for the total of all backgrounds shown as the shaded histogram. We found that choosing the cut $L_{T}>$ $2800 \mathrm{GeV}$ provides a good reach for both exclusion and discovery in this case.

Figure 16 shows the $L_{T}$ distributions for all background components, for the four best signal regions as labeled. The $L_{T}$ cut is shown in the figure as a vertical dashed line. After imposing the $L_{T}$ cut, the most important SM backgrounds are $t \bar{t} V$ and $V V V$ in the two signal regions with $2 \mathrm{SS}$ $e / \mu+\geq 1 \tau$, while the most important SM backgrounds are $W Z$ and $t \bar{t} V$ in the signal region with $\geq 2 e / \mu+2 \tau$ and $t \bar{t} h$ and $t \bar{t} V$ in the signal region with $\geq 2 e / \mu+2 \tau$ (no $Z$ ). We note that the fluctuation of about 66 normalized events in the bin from 4000 to $4500 \mathrm{GeV}$ in the lower left panel (i.e., signal region with $\geq 2 e / \mu+2 \tau$ ) of Fig. 15 is due to a single simulated event of $W Z$ background. This is because of the large cross section but extremely low yield for this background component in this signal region even after forcing decays to leptons. This is an unavoidable source of uncertainty for our analysis; given that our sample size was already $5.5 \times 10^{6}$ simulated events for this component, no practically feasible increase in sample size would yield significantly better statistics. However, in the real world the background can perhaps be determined more accurately from data.

Figure 17 shows the median expected significances for exclusion $Z_{\text {excl }}$ (left panels) and discovery $Z_{\text {disc }}$ (right panels) as a function of $M_{\tau^{\prime}}$, for $\Delta_{b} / b=0.1$ (top row), 0.2 (middle row), and 0.5 (bottom row), with the cut $L_{T}>$ $2800 \mathrm{GeV}$ imposed. The two signal regions with $2 \mathrm{SS}$ $e / \mu+\geq 1 \tau$ and the signal region with $\geq 2 e / \mu+2 \tau$ (no $Z$ ) have comparable exclusion significances and reaches. However, the latter has higher discovery significances at lower masses, as well as at higher fractional uncertainties in the background, e.g., $\Delta_{b} / b=0.5$.

Figure 17 shows a possibility of excluding doublet VLLs of masses up to about $4700 \mathrm{GeV}$ or discovering them if mass is less than about $3400 \mathrm{GeV}$ with a $70 \mathrm{TeV} p p$ collider with $30 \mathrm{ab}^{-1}$, assuming the fractional uncertainty in the background to be $\Delta_{b} / b=0.1$. If $\Delta_{b} / b=0.5$, we can still expect to exclude doublet VLLs up to $M_{\tau^{\prime}}=$ $4150 \mathrm{GeV}$ or discover them if the mass is less than about $2400 \mathrm{GeV}$. Again, a larger uncertainty in the background has a moderate effect on the prospects for exclusion, but a much larger impact on the prospects for discovery.
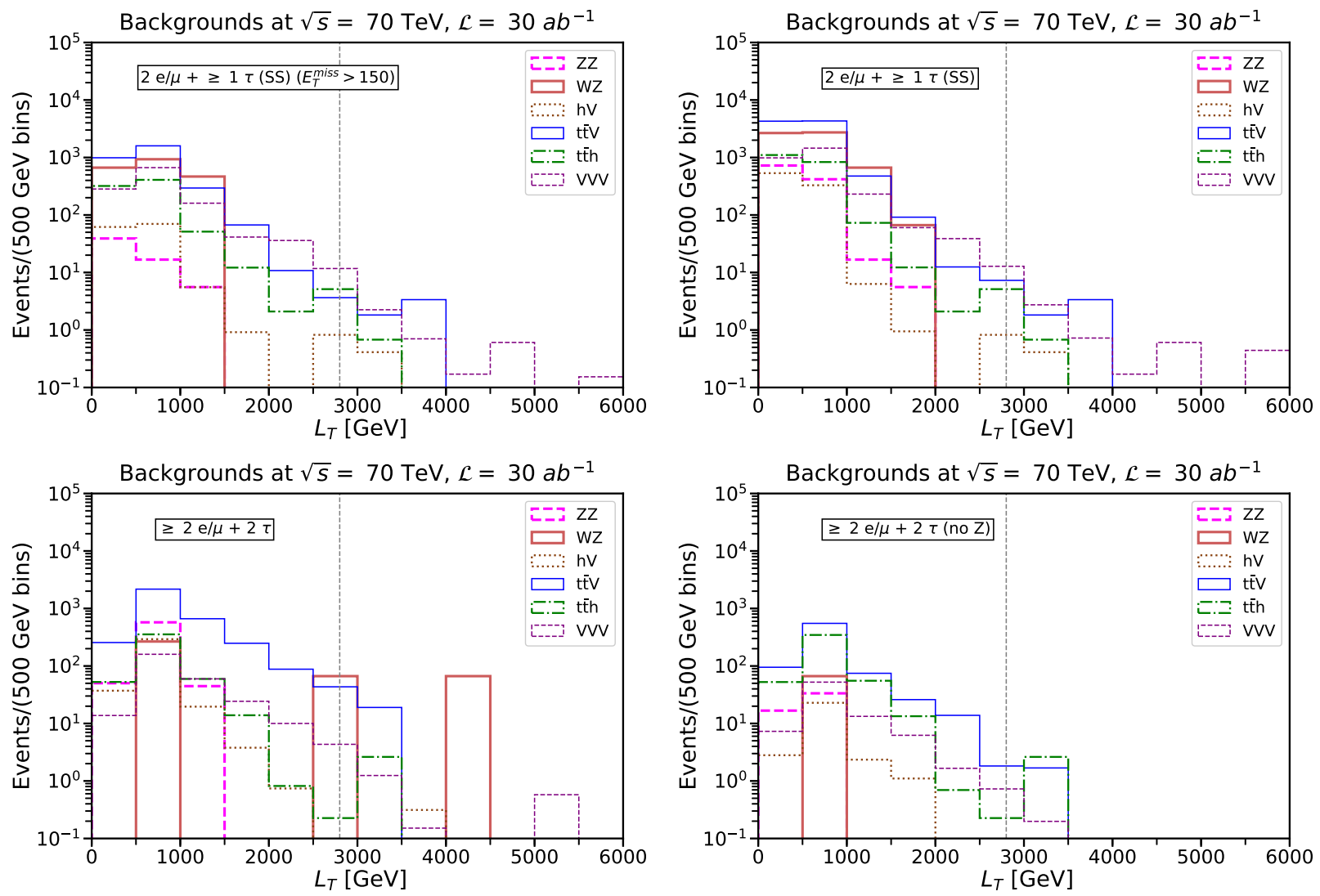

FIG. 16. $L_{T}$ event distributions for all processes contributing to total $\mathrm{SM}$ background, for $p p$ collisions at $\sqrt{s}=70 \mathrm{TeV}$ with an integrated luminosity $\mathcal{L}=30 \mathrm{ab}^{-1}$. The four panels show results for the four best signal regions, as labeled. The vertical dashed line in all four panels shows our choice of $L_{T}$ cut of $2800 \mathrm{GeV}$. 

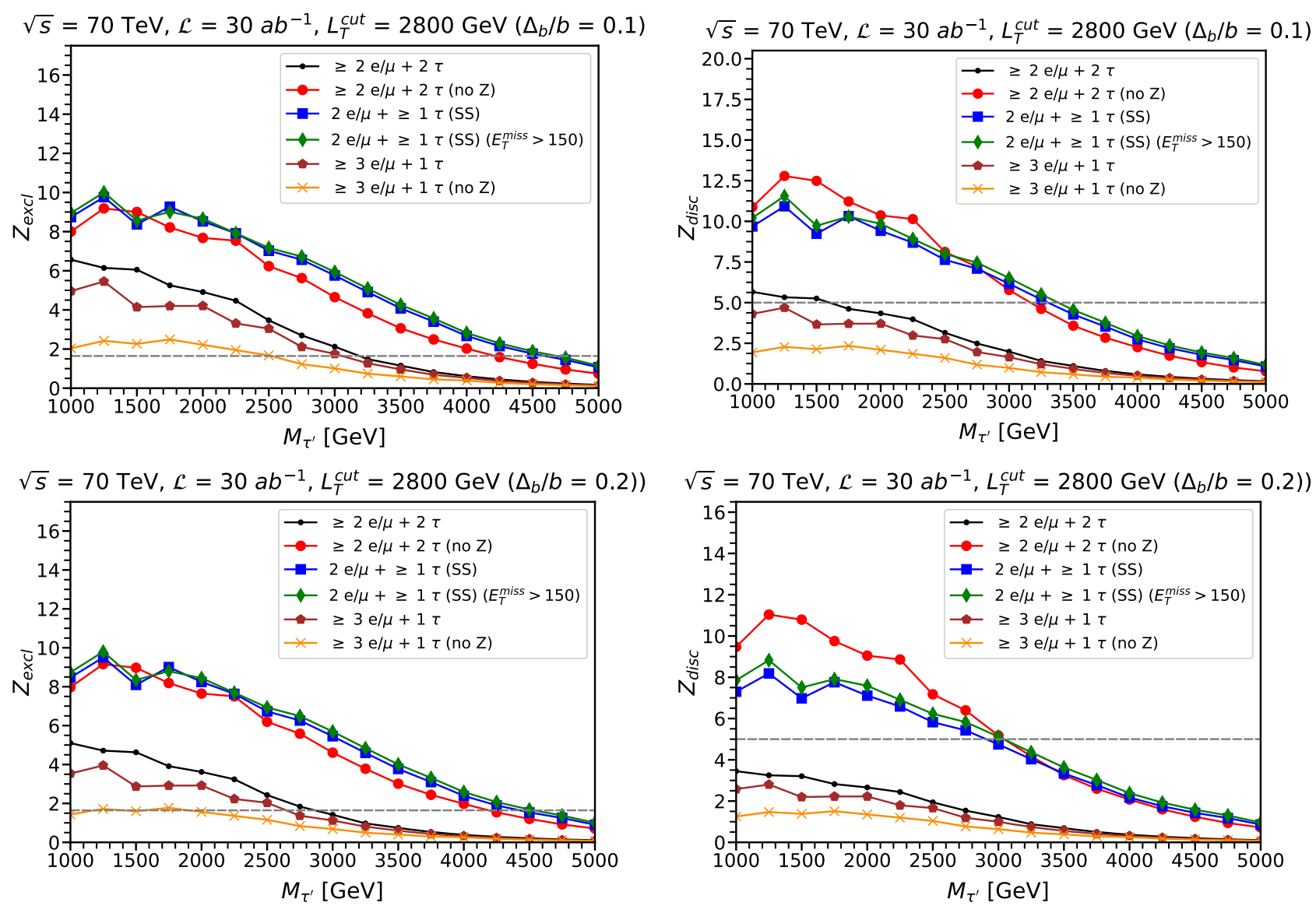

$\sqrt{s}=70 \mathrm{TeV}, \mathcal{L}=30 a b^{-1}, L_{T}^{\text {cut }}=2800 \mathrm{GeV}\left(\Delta_{b} / b=0.5\right)$

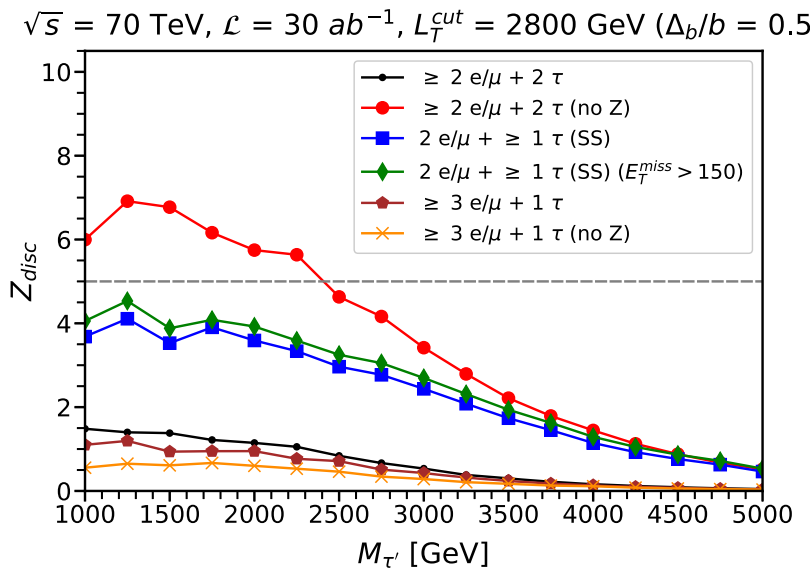

FIG. 17. The median expected significances for exclusion $Z_{\text {excl }}$ (left) and discovery $Z_{\text {disc }}$ (right) as a function of $M_{\tau^{\prime}}$ in the doublet VLL model, for $p p$ collisions at $\sqrt{s}=70 \mathrm{TeV}$ with integrated luminosity $\mathcal{L}=30 \mathrm{ab}^{-1}$, for six different signal regions as described in the text, each including a cut $L_{T}>2800 \mathrm{GeV}$. The fractional uncertainty in the background is assumed to be $\Delta_{b} / b=0.1$ (top), 0.2 (middle), and 0.5 (bottom).

\section{B. Singlet VLL models}

For $p p$ collisions with $\sqrt{s}=70 \mathrm{TeV}$ with $30 \mathrm{ab}^{-1}$, we again find that there is no possible exclusion or discovery reach for the minimal and the Higgs-philic singlet VLL models. While we find some exclusion possibility for the $Z$ philic and the $W$-phobic singlet VLL models, there are again no prospects for discovery. Figure 18 shows the resulting median expected significances for exclusion, for $\Delta_{b} / b=0.1$, for the best signal region for each of the singlet VLL models, with appropriate cuts on $L_{T}$ imposed. To approximately maximize the exclusion reaches for the $Z$-philic and $W$ phobic singlet VLL models, we chose a cut $L_{T}>2100 \mathrm{GeV}$ for the former and $L_{T}>950 \mathrm{GeV}$ for the latter. The best signal region for exclusion for the $Z$-philic singlet VLL model is the one which requires $\geq 3 e / \mu+1 \tau$, while it was $\geq 2 e / \mu+2 \tau$, no $Z$ for all other singlet VLL models. 


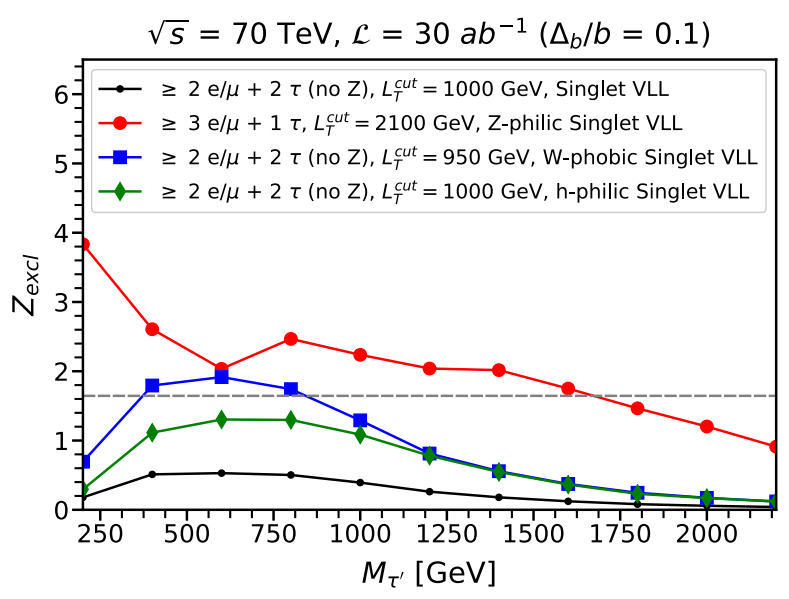

FIG. 18. The median expected significances for exclusion $Z_{\text {excl }}$ as a function of $M_{\tau^{\prime}}$ in the singlet VLL models, for $p p$ collisions at $\sqrt{s}=70 \mathrm{TeV}$ with integrated luminosity $\mathcal{L}=30 \mathrm{ab}^{-1}$, for the best signal region for each of the singlet VLL models, including a cut on $L_{T}$ as shown in the plot. The fractional uncertainty in the background is assumed to be $\Delta_{b} / b=0.1$.
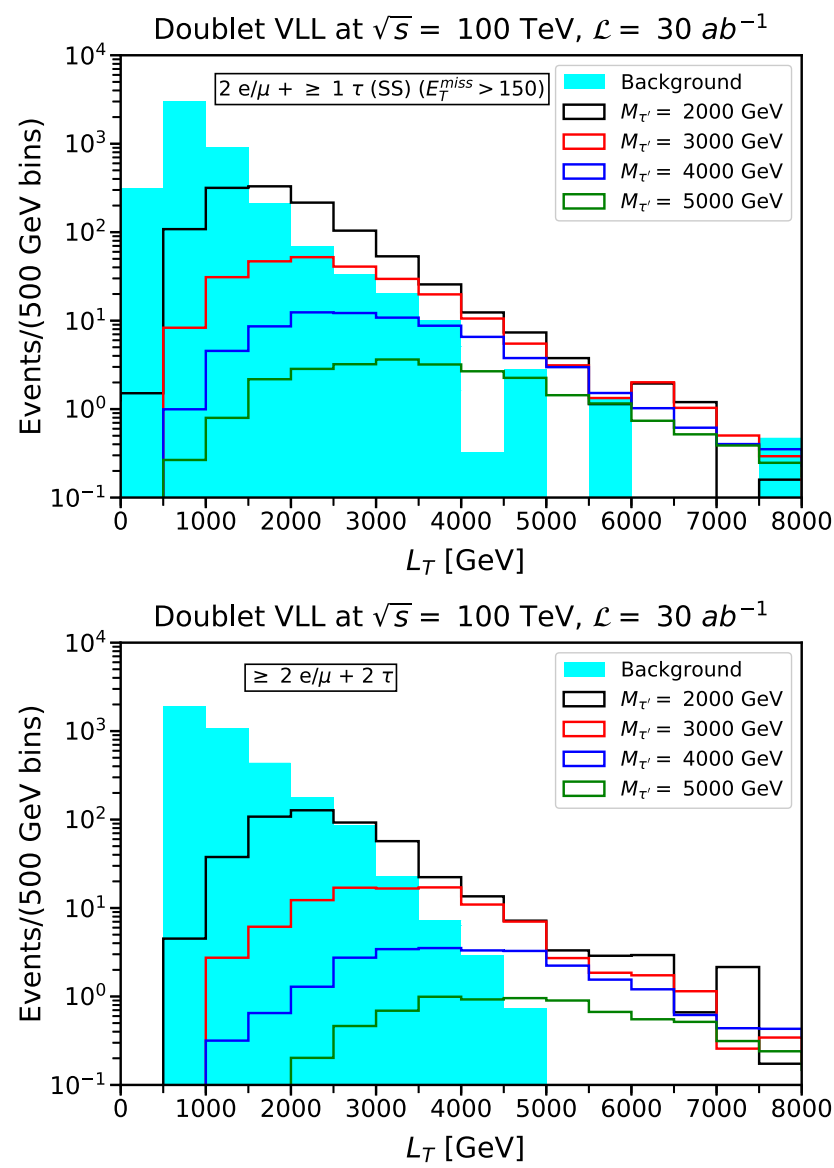

From Fig. 18, we conclude that a $70 \mathrm{TeV} p p$ collider with $30 \mathrm{ab}^{-1}$ could possibly exclude singlet VLLs with masses up to about $1700 \mathrm{GeV}$ in the $Z$-philic model or exclude masses up to $850 \mathrm{GeV}$ in the $W$-phobic model, but with no prospects for $5 \sigma$ discovery. In both minimal and the Higgs-philic singlet VLL models, there is unfortunately no possibility for exclusion or discovery, at least with the signal regions we considered.

\section{RESULTS FOR A $p p$ COLLIDER WITH $\sqrt{s}=100 \mathrm{TeV}$}

Finally, we consider the possibility of excluding or discovering VLLs at a future very high-energy $p p$ collider at $\sqrt{s}=100 \mathrm{TeV}$, with integrated luminosity of $30 \mathrm{ab}^{-1}$, in the six signal regions mentioned in Eqs. (2.29)-(2.34). We require all leptons including hadronic tau candidates to satisfy

$$
p_{T}^{\ell}>100 \mathrm{GeV}
$$
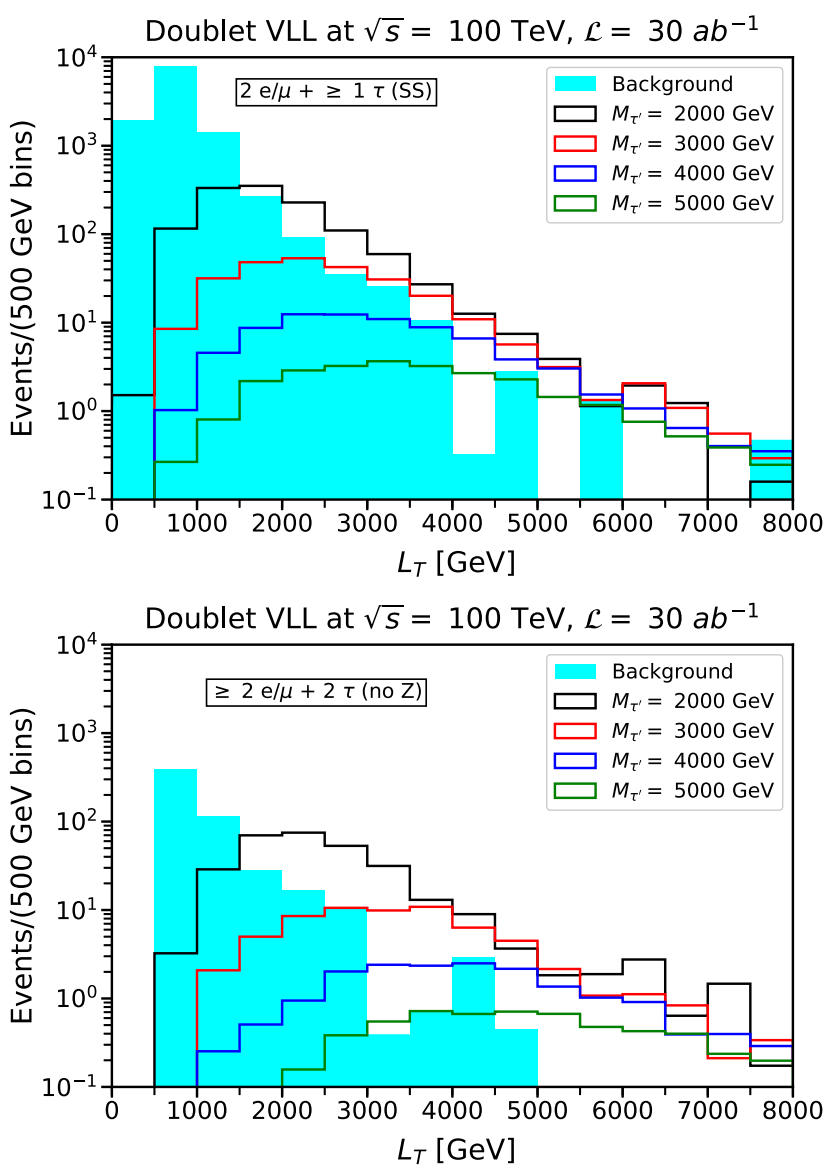

FIG. 19. $L_{T}$ event distributions for total background (shaded) and doublet VLL models (lines), for $p p$ collisions at $\sqrt{s}=100$ TeV with an integrated luminosity $\mathcal{L}=30 \mathrm{ab}^{-1}$. Four different masses $M_{\tau^{\prime}}=M_{\nu^{\prime}}=2000,3000$, 4000, and $5000 \mathrm{GeV}$ are shown in each panel. The four panels show results for the four best signal regions, as labeled. 
along with the same pseudorapidity, isolation, and other requirements of Eqs. (2.22)-(2.28). We then require the leading $e$ or $\mu$ in each event to satisfy a trigger requirement

$$
p_{T}^{e_{1}} \text { or } p_{T}^{\mu_{1}}>200 \mathrm{GeV} \text {. }
$$

\section{A. Doublet VLL model}

In Fig. 19, we show the $L_{T}$ distributions for the best four of these signal regions, for four different choices of $M_{\tau^{\prime}}$ as labeled, and for the total of all backgrounds shown as the shaded histogram. To obtain enhanced expected reaches for both exclusion and discovery, we then chose a cut $L_{T}>3500 \mathrm{GeV}$.

Figure 20 shows the $L_{T}$ distributions for all background components, for the four best signal regions as labeled. The $L_{T}$ cut is shown in the figure as a vertical dashed line. After imposing the $L_{T}$ cut, the most important SM backgrounds are $t \bar{t} V$ and $V V V$ in the two signal regions with $2 \mathrm{SS}$ $e / \mu+\geq 1 \tau$ and in the signal region with $\geq 2 e / \mu+2 \tau$, while the most important SM background in the signal region with $\geq 2 e / \mu+2 \tau$ (no $Z$ ) is $V V V$.
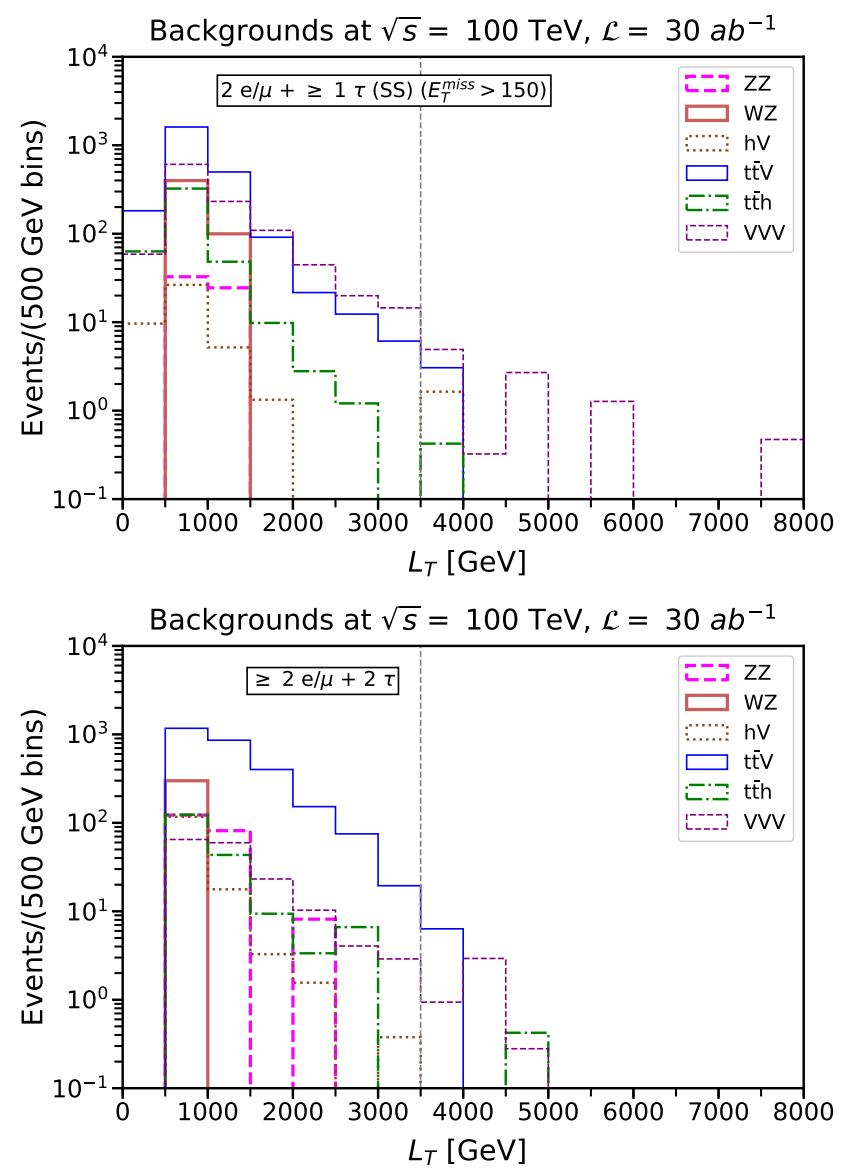

Figure 21 shows the median expected significances for exclusion $Z_{\text {excl }}$ (left panels) and discovery $Z_{\text {disc }}$ (right panels) as a function of $M_{\tau^{\prime}}$, for $\Delta_{b} / b=0.1$ (top row), 0.2 (middle row), and 0.5 (bottom row), with the cut $L_{T}>3500 \mathrm{GeV}$ imposed. The signal regions which require $2 \mathrm{SS} e / \mu+\geq 1 \tau$ usually provide the best exclusion and discovery reaches. An exception is that, with the fractional uncertainty in the background $\Delta_{b} / b=0.5$, the signal regions with $\geq 3 e / \mu+1 \tau$ have the farthest discovery reach.

From Fig. 21, we conclude that a $100 \mathrm{TeV} p p$ collider with $30 \mathrm{ab}^{-1}$ could exclude doublet VLLs with $M_{\tau^{\prime}}$ up to about $5750 \mathrm{GeV}$ or discover them if the mass is less than about $4000 \mathrm{GeV}$, assuming the fractional uncertainty in the background to be 0.1 . For $\Delta_{b} / b=0.5$, one can still expect to exclude doublet VLLs if the mass is up to about $5100 \mathrm{GeV}$ or discover them if the mass is less than about $3100 \mathrm{GeV}$. Again, as a recurring theme at all the collider options considered, a larger uncertainty in the background produces a moderate reduction of the exclusion reach, but has a much greater impact on the prospects for discovery.

We again consider the possibility of observing a mass peak for the $\tau^{\prime}$ when a clear discovery can be made.
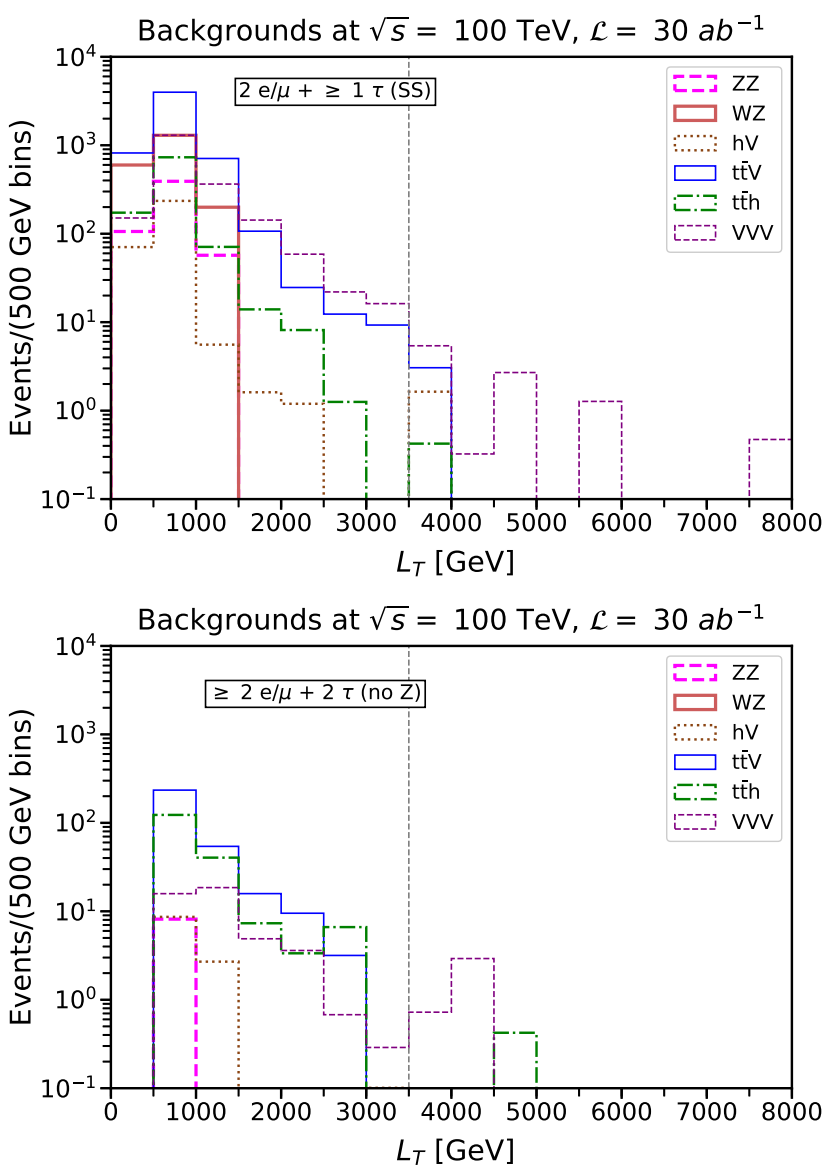

FIG. 20. $L_{T}$ event distributions for all processes contributing to total SM background, for $p p$ collisions at $\sqrt{s}=100$ TeV with an integrated luminosity $\mathcal{L}=30 \mathrm{ab}^{-1}$. The four panels show results for the four best signal regions, as labeled. The vertical dashed line in all four panels shows our choice of $L_{T}$ cut of $3500 \mathrm{GeV}$. 

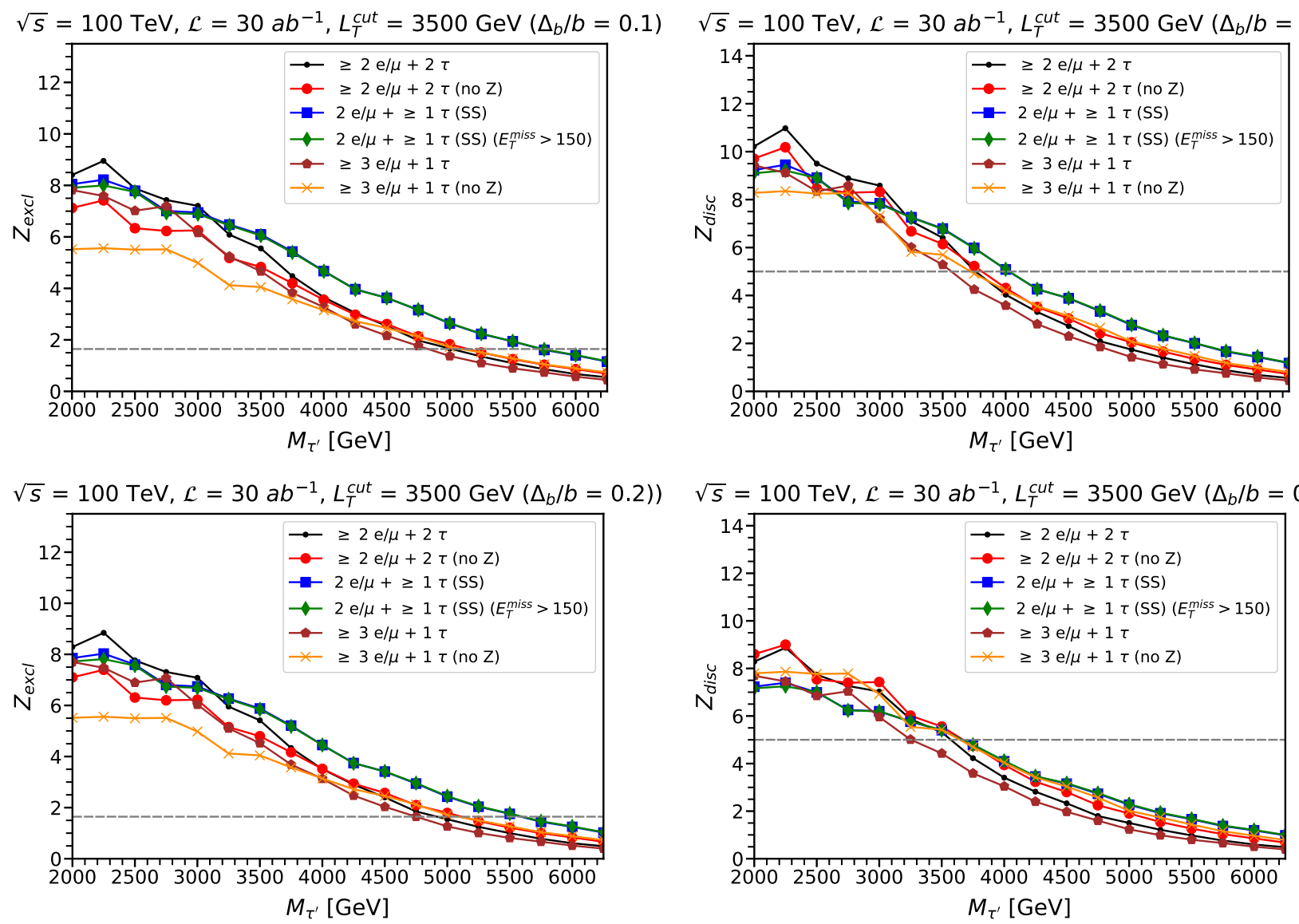

$\sqrt{s}=100 \mathrm{TeV}, \mathcal{L}=30 a b^{-1}, L_{T}^{\text {cut }}=3500 \mathrm{GeV}\left(\Delta_{b} / b=0.5\right)$
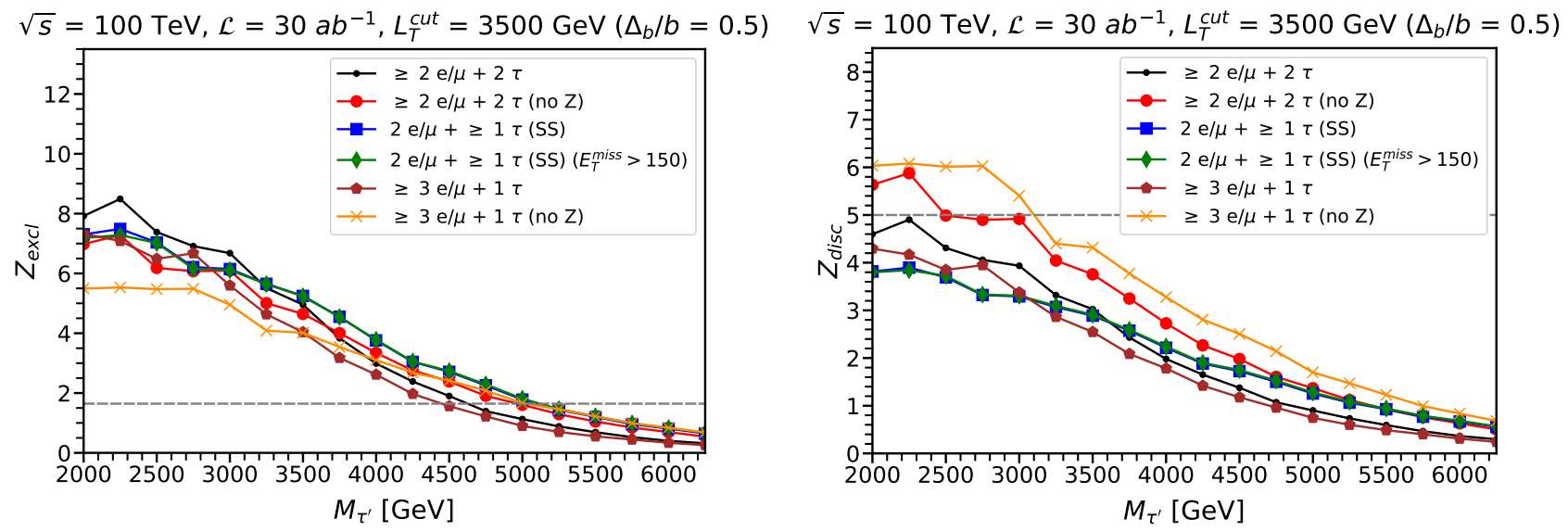

FIG. 21. The median expected significances for exclusion $Z_{\text {excl }}$ (left) and discovery $Z_{\text {disc }}$ (right) as a function of $M_{\tau^{\prime}}$ in the doublet VLL model, for $p p$ collisions at $\sqrt{s}=100 \mathrm{TeV}$ with integrated luminosity $\mathcal{L}=30 \mathrm{ab}^{-1}$, for six different signal regions as described in the text, each including a cut $L_{T}>3500 \mathrm{GeV}$. The fractional uncertainty in the background is assumed to be $\Delta_{b} / b=0.1$ (top), 0.2 (middle), and 0.5 (bottom).

Figure 22 shows the event distributions for three-body invariant mass of $\tau^{ \pm} e^{+} e^{-}$or $\tau^{ \pm} \mu^{+} \mu^{-}$, for the signal region with $\geq 2 e / \mu+2 \tau$, for three different choices of $M_{\tau^{\prime}}$, and for the total of all backgrounds shown as the shaded histogram. We require the two-body invariant mass of the $e^{+} e^{-}$or $\mu^{+} \mu^{-}$pair to be within $10 \mathrm{GeV}$ of $M_{Z}$. We also impose the cut $L_{T}>3500 \mathrm{GeV}$. From Fig. 22, we note that there are peaks in the distributions for doublet VLLs corresponding to, and slightly below, their respective masses, giving a possibility to measure the masses of doublet VLLs, if they are indeed discovered.

\section{B. Singlet VLL models}

We find no possible exclusion of singlet VLLs in the minimal and the Higgs-philic singlet VLL models, for $p p$ collisions with $\sqrt{s}=100 \mathrm{TeV}$ with integrated luminosity of $30 \mathrm{ab}^{-1}$. We find some exclusion prospects for the 


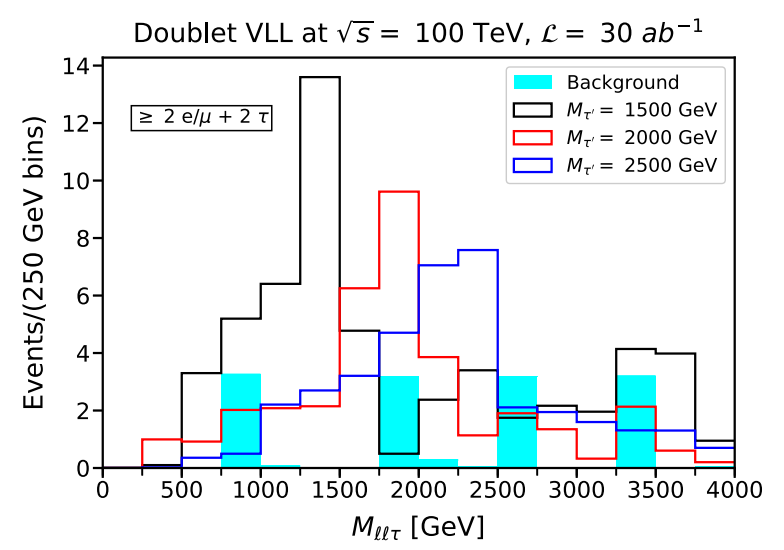

FIG. 22. The event distributions for three-body invariant masses of $\tau^{ \pm} e^{+} e^{-}$or $\tau^{ \pm} \mu^{+} \mu^{-}$, for total background (shaded) and doublet VLLs (lines), such that the $e^{+} e^{-}$or $\mu^{+} \mu^{-}$pair have an invariant mass within $10 \mathrm{GeV}$ of $M_{Z}$ in a signal region with $\geq 2 e / \mu+2 \tau$, with the cut $L_{T}>3500 \mathrm{GeV}$ imposed. Three different masses $M_{\tau^{\prime}}=M_{\nu^{\prime}}=1500,2000$, and $2500 \mathrm{GeV}$ are shown in the plot.

$Z$-philic and $W$-phobic singlet VLL models, assuming that the fractional uncertainty in the background is $\Delta_{b} / b=0.1$. Again, there are no discovery prospects in any of the singlet VLL models.

We chose cuts of $L_{T}>3000 \mathrm{GeV}$ for the $Z$-philic singlet VLL, and $L_{T}>1200 \mathrm{GeV}$ for the $W$-phobic singlet VLL, to approximately maximize the exclusion reach in each case. Figure 23 shows the resulting median expected significances for exclusion, for $\Delta_{b} / b=0.1$, for the best signal region for each of the singlet VLL models, with the cuts on $L_{T}$ imposed, as mentioned above. The best signal region for exclusion for the $Z$-philic singlet VLL model is the one which requires $\geq 3 e / \mu+1 \tau$, while it was $\geq 2 e / \mu+2 \tau$, no $Z$ for all other singlet VLL models.

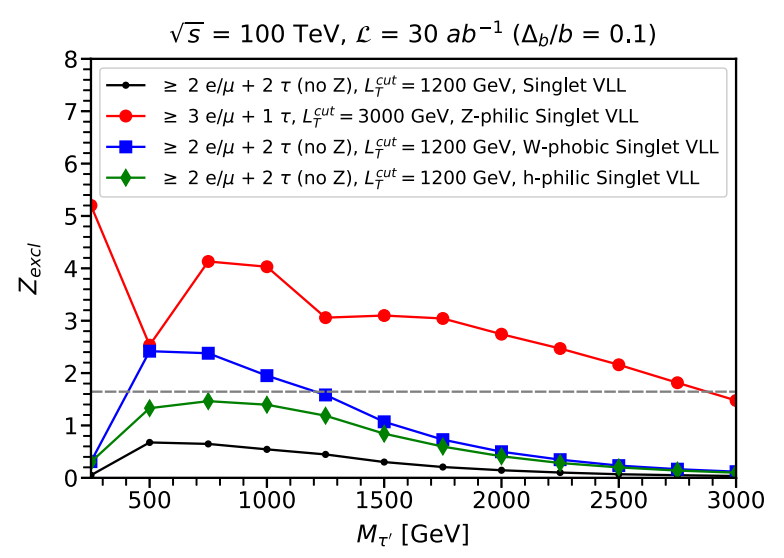

FIG. 23. The median expected significances for exclusion $Z_{\text {excl }}$ as a function of $M_{\tau^{\prime}}$ in the singlet VLL models, for $p p$ collisions at $\sqrt{s}=100 \mathrm{TeV}$ with integrated luminosity $\mathcal{L}=30 \mathrm{ab}^{-1}$, for the best signal region for each of the singlet VLL models, including a cut on $L_{T}$ as shown in the plot. The fractional uncertainty in the background is assumed to be $\Delta_{b} / b=0.1$.
TABLE I. Summary of mass reaches for the $95 \%$ exclusion and $5 \sigma$ discovery of doublet VLL at future $p p$ colliders. The fractional uncertainty in the background is assumed to be $\Delta_{b} / b=0.1$.

\begin{tabular}{lcc}
\hline \hline Collider & $\begin{array}{c}\text { Exclusion } \\
\text { reach }(\mathrm{GeV})\end{array}$ & $\begin{array}{c}\text { Discovery } \\
\text { reach }(\mathrm{GeV})\end{array}$ \\
\hline $14 \mathrm{TeV}$ HL-LHC, $3 \mathrm{ab}^{-1}$ & 1250 & 900 \\
$27 \mathrm{TeV}$ HE-LHC, $15 \mathrm{ab}^{-1}$ & 2300 & 1700 \\
$70 \mathrm{TeV} p p$ collider, $30 \mathrm{ab}^{-1}$ & 4700 & 3400 \\
$100 \mathrm{TeV} p$ p collider, $30 \mathrm{ab}^{-1}$ & 5750 & 4000 \\
\hline \hline
\end{tabular}

From Fig. 23, assuming $\Delta_{b} / b=0.1$, we conclude that a $100 \mathrm{TeV} p p$ collider with $30 \mathrm{ab}^{-1}$ should be able to exclude singlet VLLs with masses up to about $2850 \mathrm{GeV}$, if they are $Z$-philic, or exclude masses up to $1200 \mathrm{GeV}$, if they are $W$-phobic. We find that there are no prospects for exclusion of singlet VLLs in both minimal and the Higgs-philic singlet VLL models.

\section{OUTLOOK}

Vectorlike leptons are a common feature of motivated theories of physics beyond the Standard Model. In this paper, we have studied the prospects for discovering simple VLL models at future $p p$ colliders, assuming that the new particles decay promptly by mixing with taus, using multilepton signatures at high transverse momentum. The results of this paper should be considered as first estimates, which certainly should be reassessed as the plans and parameters for future colliders and their detectors become clearer. We have not attempted to make use of $h \rightarrow b \bar{b}$ decays, due in part to a relative lack of confidence in what should be assumed about $b$-jet tagging capabilities compared to $e, \mu$ identification. We have also based our analysis entirely on a cut-and-count strategy, while a more sophisticated method such as a likelihood analysis would certainly be able to do better.

Summaries of our results are shown in Table I for the doublet VLL model and in Table II for the nonminimal singlet VLL models. In the weak-isodoublet case, we have found excellent reach prospects for both $95 \%$ exclusion (if the VLLs are absent) or $5 \sigma$ discovery (if the VLLs are present). In the latter case, we also showed that there is a possibility to observe an invariant mass peak, although we did not pursue this in detail and the mass peak observation is often quite limited by statistics. In the weak-isosinglet case, the situation is much more difficult. We found no discovery or exclusion reach at all, ${ }^{5}$ at any $p p$ collider, for the simple and well-motivated (e.g., [8]) case of a promptly

\footnotetext{
${ }^{5}$ The only limits on such a particle at present are $M_{\tau^{\prime}}>$ 101.2 GeV from LEP [74], and our estimate above in a footnote in Sec. II, inferred from the ATLAS long-lived chargino search of Ref. [82], of $M_{\tau^{\prime}}>750 \mathrm{GeV}$ if it is quasistable on detector scales.
} 
TABLE II. Summary of mass reaches for the exclusion of singlet VLL in the nonminimal models at future $p p$ colliders. The fractional uncertainty in the background is assumed to be $\Delta_{b} / b=0.1$.

\begin{tabular}{|c|c|c|c|}
\hline Collider & $\begin{array}{l}\text { Z-philic } \\
(\mathrm{GeV})\end{array}$ & $\begin{array}{l}W \text {-phobic } \\
(\mathrm{GeV})\end{array}$ & $\begin{array}{l}\text { Higgs-philic } \\
(\mathrm{GeV})\end{array}$ \\
\hline $\begin{array}{l}14 \mathrm{TeV} \text { HL-LHC, } \\
3 \mathrm{ab}^{-1}\end{array}$ & 600 & 360 & 300 \\
\hline $\begin{array}{l}27 \mathrm{TeV} \text { HE-LHC, } \\
15 \mathrm{ab}^{-1}\end{array}$ & 1200 & 670 & $\ldots$ \\
\hline $\begin{array}{l}70 \mathrm{TeV} p p \text { collider, } \\
30 \mathrm{ab}^{-1}\end{array}$ & 1700 & 850 & $\ldots$ \\
\hline $\begin{array}{l}100 \mathrm{TeV} p p \text { collider, } \\
30 \mathrm{ab}^{-1}\end{array}$ & 2850 & 1200 & $\ldots$ \\
\hline
\end{tabular}

decaying pure isosinglet $\tau^{\prime}$ that mixes with the Standard Model tau. This should stand as a challenge to future work.

\section{ACKNOWLEDGMENTS}

P. N. B. thanks Olivier Mattelaer for all the helpful suggestions regarding the usage of Madgraph. P. N. B. also thanks Sergey A. Uzunyan for his kind assistance in using the NICADD compute cluster at Northern Illinois University, and Ramanpreet Singh and Elliot Parrish for technical advice. P. N. B. would also like to express special thanks to Nicholas T. Karonis, John Winans, and the entire support team of Gaea cluster at Northern Illinois University for their help and support in using their high-performance computing facility. This work was supported in part by the National Science Foundation Grant No. PHY-1719273.

\section{APPENDIX: MIXED SINGLET AND DOUBLET VLL MODELS}

Consider a generalized model framework that contains, besides the Standard Model chiral third-family leptons $\ell_{3}$ and $\bar{e}_{3}$, a vectorlike weak-isosinglet pair $E, \bar{E}$ and a weakisodoublet pair $L, \bar{L}$. (Here we use two-component fermion notation $[90,91]$, so that barred fields have electric charge +1 and unbarred fields have electric charge -1 . The bar is part of the name of the two-component fermion field and does not denote any kind of conjugation.) After electroweak symmetry breaking, the Lagrangian mass terms for the charged leptons can be written in the form

$$
-\mathcal{L}=\left(\begin{array}{lll}
\bar{e}_{3} & \bar{E} & \bar{L}
\end{array}\right) \mathcal{M}\left(\begin{array}{c}
\ell_{3} \\
E \\
L
\end{array}\right)+\text { c.c. },
$$

where the charged lepton mixing mass matrix is

$$
\mathcal{M}=\left(\begin{array}{ccc}
y_{\tau} v & 0 & \epsilon_{2} v \\
\epsilon_{1} v & M_{1} & x_{2} v \\
0 & x_{1} v & M_{2}
\end{array}\right)
$$

where $v=174 \mathrm{GeV}$ is the Standard Model Higgs expectation value, $y_{\tau}, \epsilon_{1}, \epsilon_{2}, x_{1}$, and $x_{2}$ are Yukawa couplings, and $M_{1}$ and $M_{2}$ are electroweak-singlet bare mass terms for the isosinglet and isodoublet vectorlike lepton pairs, respectively. This can be diagonalized to obtain mass eigenstates according to

$$
R^{*} \mathcal{M} L^{\dagger}=\operatorname{diag}\left(M_{\tau}, M_{\tau^{\prime}}, M_{\tau^{\prime \prime}}\right)
$$

where $R$ and $L$ are unitary $3 \times 3$ matrices and, by convention, $M_{\tau}<M_{\tau^{\prime}}<M_{\tau^{\prime \prime}}$, where $\tau$ is the usual tau lepton with $M_{\tau}=1.777 \mathrm{GeV}$, and in the special cases considered here $\tau^{\prime \prime}$ will be taken to be heavy enough to decouple from direct experimental observation. The neutral VLL $\nu^{\prime}$ has mass $M_{2}$.

For the lighter new charged VLL $\tau^{\prime}$, we have partial decay widths

$\Gamma\left(\tau^{\prime} \rightarrow W \nu_{\tau}\right)=\frac{M_{\tau^{\prime}}}{32 \pi}\left(1-r_{W}\right)^{2}\left(1+2 r_{W}\right)\left|g_{\tau^{\prime} \nu_{\tau}^{\dagger}}^{W}\right|^{2} / r_{W}$,

$$
\begin{aligned}
& \Gamma\left(\tau^{\prime} \rightarrow Z \tau\right) \\
& =\frac{M_{\tau^{\prime}}}{32 \pi}\left(1-r_{Z}\right)^{2}\left(1+2 r_{Z}\right)\left(\left|g_{\tau^{\prime} \tau^{\dagger}}^{Z}\right|^{2}+\left|g_{\bar{\tau}^{\prime} \bar{\tau}^{\dagger}}^{Z}\right|^{2}\right) / r_{Z}, \\
& \Gamma\left(\tau^{\prime} \rightarrow h \tau\right)=\frac{M_{\tau^{\prime}}}{32 \pi}\left(1-r_{h}\right)^{2}\left(\left|y_{\tau^{\prime} \bar{\tau}}^{h}\right|^{2}+\left|y_{\tau^{\tau^{\prime}}}^{h}\right|^{2}\right),
\end{aligned}
$$

where $r_{X}=M_{X}^{2} / M_{\tau^{\prime}}^{2}$ for each of $X=h, Z, W$, and

$$
\begin{gathered}
g_{\tau_{j} \nu_{\tau}^{\dagger}}^{W}=\frac{g}{\sqrt{2}} L_{j 1}^{*}, \\
g_{\tau_{j} \tau_{k}^{*}}^{Z}=\frac{g}{\cos \theta_{W}}\left[\frac{1}{2} L_{j 2}^{*} L_{k 2}+\left(-\frac{1}{2}+\sin ^{2} \theta_{W}\right) \delta_{j k}\right], \\
g_{\bar{\tau}_{j} \tau_{k}^{*}}^{Z}=\frac{g}{\cos \theta_{W}}\left[\frac{1}{2} R_{j 3}^{*} R_{k 3}-\sin ^{2} \theta_{W} \delta_{j k}\right], \\
y_{\tau_{j} \bar{\tau}_{k}}^{h}=\frac{1}{\sqrt{2}}\left[y_{\tau} L_{j 1}^{*} R_{k 1}^{*}+\epsilon_{2} L_{j 3}^{*} R_{k 1}^{*}+\epsilon_{1} L_{j 1}^{*} R_{k 2}^{*}\right. \\
\left.+x_{2} L_{j 3}^{*} R_{k 2}^{*}+x_{1} L_{j 2}^{*} R_{k 3}^{*}\right],
\end{gathered}
$$

for $\left(\tau_{1}, \tau_{2}, \tau_{3}\right)$ corresponding to mass eigenstates $\left(\tau, \tau^{\prime}, \tau^{\prime \prime}\right)$, respectively.

In general, the decay widths to Standard Model states are quadratic in $\epsilon_{1}, \epsilon_{2}$ for small values of those parameters. For example, expanding to obtain the terms proportional to $\epsilon_{1}^{2}$, $\epsilon_{1} \epsilon_{2}$, and $\epsilon_{2}^{2}$, and then keeping only the leading order in a further expansion in $y_{\tau}, x_{1}$, and $x_{2}$ in each of these terms, we obtain for $M_{2}^{2} \ll M_{1}^{2}$, 


$$
\begin{aligned}
& \left|g_{\tau^{\prime} \nu_{\tau}^{\dagger}}^{W}\right|^{2} / r_{W} \approx\left[\frac{\epsilon_{1} v\left(x_{1} M_{1}+x_{2} M_{2}\right)}{M_{1}^{2}}-\frac{\epsilon_{2} y_{\tau} v}{M_{2}}\right]^{2}, \\
& \left(\left|g_{\tau^{\prime} \tau^{\dagger}}^{Z}\right|^{2}+\left|g_{\bar{\tau}^{\prime} \bar{\tau}^{\dagger}}^{Z}\right|^{2}\right) / r_{Z} \approx \epsilon_{1}^{2} v^{2} \frac{M_{2}^{2}\left(x_{1} M_{2}+x_{2} M_{1}\right)^{2}}{2 M_{1}^{6}} \\
& -\epsilon_{1} \epsilon_{2} y_{\tau} v^{2} \frac{\left(x_{1} M_{1}+x_{2} M_{2}\right)}{M_{1}^{2} M_{2}}+\frac{\epsilon_{2}^{2}}{2}, \\
& \left|y_{\tau^{\prime} \bar{\tau}}^{h}\right|^{2}+\left|y_{\tau \bar{\tau}^{\prime}}^{h}\right|^{2} \approx \epsilon_{1}^{2} v^{2} \frac{\left(2 x_{1} M_{1}+x_{2} M_{2}\right)^{2}}{2 M_{1}^{4}} \\
& -3 \epsilon_{1} \epsilon_{2} y_{\tau} v^{2} \frac{\left(x_{1} M_{1}+x_{2} M_{2}\right)}{M_{1}^{2} M_{2}}+\frac{\epsilon_{2}^{2}}{2},
\end{aligned}
$$

and for $M_{1}^{2} \ll M_{2}^{2}$,

$$
\begin{gathered}
\left|g_{\tau^{\prime} \nu_{\tau}^{\dagger}}^{W}\right|^{2} / r_{W} \approx\left[\epsilon_{1}-\epsilon_{2} y_{\tau} v^{2} \frac{\left(x_{1} M_{2}+x_{2} M_{1}\right)}{M_{1} M_{2}^{2}}\right]^{2}, \\
\left(\left|g_{\tau^{\prime} \tau^{\dagger}}^{Z}\right|^{2}+\left|g_{\bar{\tau}^{\prime} \bar{\tau}^{\dagger}}^{Z}\right|^{2}\right) / r_{Z} \approx \\
+\epsilon_{1}^{2}-\epsilon_{1} \epsilon_{2} y_{\tau} v^{2} \frac{\left(x_{1} M_{2}+x_{2} M_{1}\right)}{M_{1} M_{2}^{2}} \\
\left.\left|y_{\tau^{\prime} \bar{\tau}}\right|^{2}+\mid y_{1}+x_{2} M_{2}\right)^{2} \\
2 M_{2}^{6}
\end{gathered}
$$

Some special limits of interest follow:

(1) If $M_{2}^{2} \ll M_{1}^{2}$ and $\epsilon_{1}=0$, then the isosinglet heavier fermion mass eigenstate $\tau^{\prime \prime}$ decouples from experiment, and the lighter states $\tau^{\prime}$ and $\nu^{\prime}$ form the minimal doublet VLL model as discussed above in Sec. II with $\epsilon=\epsilon_{2}$ and $M_{\tau^{\prime}}=M_{\nu^{\prime}}=M_{2}$, and branching ratios that asymptotically approach $\operatorname{BR}\left(\tau^{\prime} \rightarrow h \tau\right)=\operatorname{BR}\left(\tau^{\prime} \rightarrow Z \tau\right)=0.5$ and $\operatorname{BR}\left(\nu^{\prime} \rightarrow W \tau\right)=1$.

(2) If $M_{1}^{2} \ll M_{2}^{2}$ and $\epsilon_{2}=0$, then the heavy isodoublet fermions $\tau^{\prime \prime}$ and $\nu^{\prime}$ decouple, and the result is the minimal singlet VLL model as discussed above in Sec. II with $\epsilon=\epsilon_{1}$ and $M_{\tau^{\prime}}=M_{1}$, and branching ratios that asymptotically approach $\operatorname{BR}\left(\tau^{\prime} \rightarrow h \tau\right)=$ $\mathrm{BR}\left(\tau^{\prime} \rightarrow Z \tau\right)=0.25$ and $\mathrm{BR}\left(\tau^{\prime} \rightarrow W \nu_{\tau}\right)=0.5$.

(3) If $M_{1}^{2} \ll M_{2}^{2}$ and $\epsilon_{1}=0$ and $\epsilon_{2} \neq 0$, then the lightest new fermion will again be a mostly isosinglet $\tau^{\prime}$ vectorlike lepton with mass approximately $M_{\tau^{\prime}}=$ $M_{1}$. Since the $\tau^{\prime}$ is mostly isosinglet, its production cross section is nearly the same as in the minimal singlet VLL model. However, due to its mixing with the heavier isodoublets rather than direct mixing with the Standard Model tau lepton, the decay $\tau^{\prime} \rightarrow$ $W \nu_{\tau}$ is highly suppressed. The possibilities include the following subcases:

(a) $W$-phobic singlet VLL: if $x_{1}=0$, then $\operatorname{BR}\left(\tau^{\prime} \rightarrow h \tau\right) \approx \operatorname{BR}\left(\tau^{\prime} \rightarrow Z \tau\right) \approx 0.5$.

(b) Higgs-philic singlet VLL: if $x_{2}=0$, then $\operatorname{BR}\left(\tau^{\prime} \rightarrow h \tau\right) \approx 1$.

(c) Z-philic singlet VLL: if $x_{1} \approx-x_{2} M_{1} / 2 M_{2}$, then $\operatorname{BR}\left(\tau^{\prime} \rightarrow Z \tau\right) \approx 1$.

These of course do not exhaust the possibilities, and in a more general search it would be sensible to simply take $\Gamma\left(\tau^{\prime} \rightarrow Z \tau\right)$ and $\Gamma\left(\tau^{\prime} \rightarrow h \tau\right)$ and $\Gamma\left(\tau^{\prime} \rightarrow W \nu_{\tau}\right)$ to be free parameters.
[1] G. Aad et al. (ATLAS Collaboration), Observation of a new particle in the search for the Standard Model Higgs boson with the ATLAS detector at the LHC, Phys. Lett. B 716, 1 (2012); S. Chatrchyan et al. (CMS Collaboration), Observation of a new boson at a mass of $125 \mathrm{GeV}$ with the CMS experiment at the LHC, Phys. Lett. B 716, 30 (2012).

[2] See, for example, A. Djouadi and A. Lenz, Sealing the fate of a fourth generation of fermions, Phys. Lett. B 715, 310 (2012); A. Lenz, Constraints on a fourth generation of fermions from Higgs Boson searches, Adv. High Energy Phys. 2013, 910275 (2013); O. Eberhardt, G. Herbert, H. Lacker, A. Lenz, A. Menzel, U. Nierste, and M. Wiebusch, Impact of a Higgs Boson at a Mass of $126 \mathrm{GeV}$ on the
Standard Model with Three and Four Fermion Generations, Phys. Rev. Lett. 109, 241802 (2012), and references therein.

[3] For a review, see S. P. Martin, A supersymmetry primer, Adv. Ser. Dir. High Energy Phys. 21, 1 (2010); 18, 1 (1998).

[4] T. Moroi and Y. Okada, Radiative corrections to Higgs masses in the supersymmetric model with an extra family and antifamily, Mod. Phys. Lett. A 07, 187 (1992).

[5] T. Moroi and Y. Okada, Upper bound of the lightest neutral Higgs mass in extended supersymmetric Standard Models, Phys. Lett. B 295, 73 (1992).

[6] K. S. Babu, I. Gogoladze, and C. Kolda, Perturbative unification and Higgs boson mass bounds, arXiv:hep-ph/ 0410085 . 
[7] K. S. Babu, I. Gogoladze, M. U. Rehman, and Q. Shafi, Higgs Boson mass, sparticle spectrum and little hierarchy problem in extended MSSM, Phys. Rev. D 78, 055017 (2008).

[8] S. P. Martin, Extra vector-like matter and the lightest Higgs scalar boson mass in low-energy supersymmetry, Phys. Rev. D 81, 035004 (2010).

[9] P. W. Graham, A. Ismail, S. Rajendran, and P. Saraswat, A little solution to the little hierarchy problem: A vector-like generation, Phys. Rev. D 81, 055016 (2010).

[10] S. P. Martin, Raising the Higgs mass with Yukawa couplings for isotriplets in vector-like extensions of minimal supersymmetry, Phys. Rev. D 82, 055019 (2010).

[11] M. Endo, K. Hamaguchi, S. Iwamoto, and N. Yokozaki, Higgs mass and muon anomalous magnetic moment in supersymmetric models with vector-like matters, Phys. Rev. D 84, 075017 (2011).

[12] J. L. Evans, M. Ibe, and T. T. Yanagida, Probing extra matter in gauge mediation through the lightest Higgs Boson mass, arXiv:1108.3437.

[13] T. Li, J. A. Maxin, D. V. Nanopoulos, and J. W. Walker, A Higgs mass shift to $125 \mathrm{GeV}$ and a multi-jet supersymmetry signal: Miracle of the Flippons at the $\sqrt{s}=7 \mathrm{TeV}$ LHC, Phys. Lett. B 710, 207 (2012).

[14] T. Moroi, R. Sato, and T. T. Yanagida, Extra matters decree the relatively heavy Higgs of mass about $125 \mathrm{GeV}$ in the supersymmetric model, Phys. Lett. B 709, 218 (2012).

[15] M. Endo, K. Hamaguchi, S. Iwamoto, and N. Yokozaki, Higgs mass, muon $\mathrm{g}-2$, and LHC prospects in gauge mediation models with vector-like matters, Phys. Rev. D 85, 095012 (2012).

[16] M. Endo, K. Hamaguchi, S. Iwamoto, and N. Yokozaki, Vacuum stability bound on extended GMSB models, J. High Energy Phys. 06 (2012) 060.

[17] K. Nakayama and N. Yokozaki, Peccei-Quinn extended gauge-mediation model with vector-like matter, J. High Energy Phys. 11 (2012) 158.

[18] S. P. Martin and J. D. Wells, Implications of gauge-mediated supersymmetry breaking with vector-like quarks and a 125 GeV Higgs boson, Phys. Rev. D 86, 035017 (2012).

[19] A. M. Sirunyan et al. (CMS Collaboration), Search for vectorlike $\mathrm{T}$ and $\mathrm{B}$ quark pairs in final states with leptons at $\sqrt{s}=13 \mathrm{TeV}$, J. High Energy Phys. 08 (2018) 177; Search for top quark partners with charge $5 / 3$ in the same-sign dilepton and single-lepton final states in proton-proton collisions at $\sqrt{s}=13 \mathrm{TeV}$, J. High Energy Phys. 03 (2019) 082.

[20] M. Aaboud et al. (ATLAS Collaboration), Search for pair production of vector-like top quarks in events with one lepton, jets, and missing transverse momentum in $\sqrt{\mathrm{s}}=$ $13 \mathrm{TeV} p p$ collisions with the ATLAS detector, J. High Energy Phys. 08 (2017) 052; Search for pair production of heavy vector-like quarks decaying to high- $\mathrm{p}_{T} \mathrm{~W}$ bosons and $\mathrm{b}$ quarks in the lepton-plus-jets final state in pp collisions at $\sqrt{s}=13 \mathrm{TeV}$ with the ATLAS detector, J. High Energy Phys. 10 (2017) 141; Search for pair production of up-type vector-like quarks and for four-top-quark events in final states with multiple $b$-jets with the ATLAS detector, J. High Energy Phys. 07 (2018) 089; Search for pair production of heavy vector-like quarks decaying into high- $p_{T} W$ bosons and top quarks in the lepton-plus-jets final state in $p p$ collisions at $\sqrt{s}=13 \mathrm{TeV}$ with the ATLAS detector, J. High Energy Phys. 08 (2018) 048; Search for pair- and single-production of vector-like quarks in final states with at least one $Z$ boson decaying into a pair of electrons or muons in $p p$ collision data collected with the ATLAS detector at $\sqrt{s}=13 \mathrm{TeV}$, arXiv:1806.10555; Search for pair production of heavy vector-like quarks decaying into hadronic final states in $p p$ collisions at $\sqrt{s}=13 \mathrm{TeV}$ with the ATLAS detector, Phys. Rev. D 98, 092005 (2018); Combination of the Searches for Pair-Produced Vector-Like Partners of the Third-Generation Quarks at $\sqrt{s}=13 \mathrm{TeV}$ with the ATLAS Detector, Phys. Rev. Lett. 121, 211801 (2018).

[21] S. Dimopoulos, N. Tetradis, R. Esmailzadeh, and L. J. Hall, TeV dark matter, Nucl. Phys. B349, 714 (1991).

[22] M. Sher, Charged leptons with nanosecond lifetimes, Phys. Rev. D 52, 3136 (1995).

[23] S. D. Thomas and J. D. Wells, Phenomenology of Massive Vectorlike Doublet Leptons, Phys. Rev. Lett. 81, 34 (1998).

[24] P. H. Frampton, P. Q. Hung, and M. Sher, Quarks and leptons beyond the third generation, Phys. Rep. 330, 263 (2000).

[25] F. del Aguila, J. de Blas, and M. Perez-Victoria, Effects of new leptons in electroweak precision data, Phys. Rev. D 78, 013010 (2008).

[26] F. del Aguila, A. Carmona, and J. Santiago, Neutrino masses from an A4 symmetry in holographic composite Higgs models, J. High Energy Phys. 08 (2010) 127; Tau Custodian searches at the LHC, Phys. Lett. B 695, 449 (2011); A. Carmona and F. Goertz, Custodial leptons and Higgs decays, J. High Energy Phys. 04 (2013) 163.

[27] K. Ishiwata and M. B. Wise, Higgs Properties and fourth generation leptons, Phys. Rev. D 84, 055025 (2011).

[28] R. Dermisek, Insensitive unification of gauge couplings, Phys. Lett. B 713, 469 (2012).

[29] A. Joglekar, P. Schwaller, and C. E. M. Wagner, Dark matter and enhanced Higgs to Di-photon rate from vector-like leptons, J. High Energy Phys. 12 (2012) 064.

[30] J. Kearney, A. Pierce, and N. Weiner, Vectorlike Fermions and Higgs couplings, Phys. Rev. D 86, 113005 (2012).

[31] C. Arina, R. N. Mohapatra, and N. Sahu, Co-genesis of matter and dark matter with vector-like fourth generation leptons, Phys. Lett. B 720, 130 (2013).

[32] B. Batell, S. Jung, and H. M. Lee, Singlet assisted vacuum stability and the Higgs to diphoton rate, J. High Energy Phys. 01 (2013) 135.

[33] R. Dermisek, Unification of gauge couplings in the standard model with extra vectorlike families, Phys. Rev. D 87, 055008 (2013).

[34] W. Z. Feng and P. Nath, Higgs diphoton rate and mass enhancement with vectorlike leptons and the scale of supersymmetry, Phys. Rev. D 87, 075018 (2013).

[35] A. Joglekar, P. Schwaller, and C. E. M. Wagner, A supersymmetric theory of vector-like leptons, J. High Energy Phys. 07 (2013) 046.

[36] P. Schwaller, T. M. P. Tait, and R. Vega-Morales, Dark matter and vectorlike leptons from gauged lepton number, Phys. Rev. D 88, 035001 (2013).

[37] R. Dermisek and A. Raval, Explanation of the Muon g-2 anomaly with vectorlike leptons and its implications for Higgs decays, Phys. Rev. D 88, 013017 (2013). 
[38] K. Ishiwata and M. B. Wise, Phenomenology of heavy vectorlike leptons, Phys. Rev. D 88, 055009 (2013).

[39] M. Fairbairn and P. Grothaus, Baryogenesis and dark matter with vector-like Fermions, J. High Energy Phys. 10 (2013) 176.

[40] W. Altmannshofer, M. Bauer, and M. Carena, Exotic leptons: Higgs, flavor and collider phenomenology, J. High Energy Phys. 01 (2014) 060.

[41] S. Fichet, G. von Gersdorff, O. Kepka, B. Lenzi, C. Royon, and M. Saimpert, Probing new physics in diphoton production with proton tagging at the Large Hadron Collider, Phys. Rev. D 89, 114004 (2014); S. Fichet, G. von Gersdorff, B. Lenzi, C. Royon, and M. Saimpert, Light-by-light scattering with intact protons at the LHC: From Standard Model to new physics, J. High Energy Phys. 02 (2015) 165.

[42] A. Falkowski, D. M. Straub, and A. Vicente, Vector-like leptons: Higgs decays and collider phenomenology, J. High Energy Phys. 05 (2014) 092.

[43] J. Halverson, N. Orlofsky, and A. Pierce, Vectorlike leptons as the tip of the dark matter Iceberg, Phys. Rev. D 90, 015002 (2014).

[44] B. A. Dobrescu and C. Frugiuele, Hidden GeV-Scale Interactions of Quarks, Phys. Rev. Lett. 113, 061801 (2014).

[45] S. A. R. Ellis, R. M. Godbole, S. Gopalakrishna, and J. D. Wells, Survey of vector-like fermion extensions of the Standard Model and their phenomenological implications, J. High Energy Phys. 09 (2014) 130.

[46] A. Falkowski and R. Vega-Morales, Exotic Higgs decays in the golden channel, J. High Energy Phys. 12 (2014) 037.

[47] B. Holdom and M. Ratzlaff, Neglected heavy leptons at the LHC, Phys. Rev. D 90, 013015 (2014).

[48] R. Dermisek, A. Raval, and S. Shin, Effects of vectorlike leptons on $h \rightarrow 4 \ell$ and the connection to the muon g-2 anomaly, Phys. Rev. D 90, 034023 (2014).

[49] B. A. Dobrescu and A. Martin, Interpretations of anomalous LHC events with electrons and jets, Phys. Rev. D 91, 035019 (2015).

[50] R. Dermisek, J. P. Hall, E. Lunghi, and S. Shin, Limits on vectorlike leptons from searches for anomalous production of multi-lepton events, J. High Energy Phys. 12 (2014) 013.

[51] B. Holdom and M. Ratzlaff, Distinctive heavy Higgs boson decays, Phys. Rev. D 91, 035031 (2015).

[52] G. Aad et al. (ATLAS Collaboration), Search for heavy lepton resonances decaying to a $Z$ boson and a lepton in $p p$ collisions at $\sqrt{s}=8 \mathrm{TeV}$ with the ATLAS detector, J. High Energy Phys. 09 (2015) 108.

[53] K. Ishiwata, Z. Ligeti, and M. B. Wise, New vector-like fermions and flavor physics, J. High Energy Phys. 10 (2015) 027.

[54] N. Bizot and M. Frigerio, Fermionic extensions of the Standard Model in light of the Higgs couplings, J. High Energy Phys. 01 (2016) 036.

[55] R. Dermisek, E. Lunghi, and S. Shin, Two Higgs doublet model with vectorlike leptons and contributions to $p p \rightarrow$ $W W$ and $H \rightarrow W W$, J. High Energy Phys. 02 (2016) 119.

[56] S. Bhattacharya, N. Sahoo, and N. Sahu, Minimal vectorlike leptonic dark matter and signatures at the LHC, Phys. Rev. D 93, 115040 (2016).
[57] N. Kumar and S. P. Martin, Vectorlike leptons at the large hadron collider, Phys. Rev. D 92, 115018 (2015).

[58] R. Dermisek, E. Lunghi, and S. Shin, New decay modes of heavy Higgs bosons in a two Higgs doublet model with vectorlike leptons, J. High Energy Phys. 05 (2016) 148.

[59] M. Abdullah, J. L. Feng, S. Iwamoto, and B. Lillard, Heavy bino dark matter and collider signals in the MSSM with vectorlike fourth-generation particles, Phys. Rev. D 94, 095018 (2016).

[60] R. Dermisek, E. Lunghi, and S. Shin, New constraints and discovery potential for Higgs to Higgs cascade decays through vectorlike leptons, J. High Energy Phys. 10 (2016) 081.

[61] J. L. Feng, B. Fornal, I. Galon, S. Gardner, J. Smolinsky, T. M. P. Tait, and P. Tanedo, Particle physics models for the $17 \mathrm{MeV}$ anomaly in beryllium nuclear decays, Phys. Rev. D 95, 035017 (2017).

[62] A. Angelescu and G. Arcadi, Dark matter phenomenology of SM and enlarged Higgs sectors extended with vector like leptons, Eur. Phys. J. C 77, 456 (2017).

[63] S. Bahrami, M. Frank, D. K. Ghosh, N. Ghosh, and I. Saha, Dark matter and collider studies in the left-right symmetric model with vectorlike leptons, Phys. Rev. D 95, 095024 (2017).

[64] A. Choudhury, L. Darme, L. Roszkowski, E. M. Sessolo, and S. Trojanowski, Muon $g-2$ and related phenomenology in constrained vector-like extensions of the MSSM, J. High Energy Phys. 05 (2017) 072.

[65] Z. Poh and S. Raby, Vectorlike leptons: Muon g-2 anomaly, lepton flavor violation, Higgs boson decays, and lepton nonuniversality, Phys. Rev. D 96, 015032 (2017).

[66] Q. Lu, D. E. Morrissey, and A. M. Wijangco, Higgs boson decays to dark photons through the vectorized lepton portal, J. High Energy Phys. 06 (2017) 138.

[67] J. Y. Araz, S. Banerjee, M. Frank, B. Fuks, and A. Goudelis, Dark matter and collider signals in an MSSM extension with vector-like multiplets, Phys. Rev. D 98, 115009 (2018).

[68] N. F. Bell, M. J. Dolan, L. S. Friedrich, M. J. RamseyMusolf, and R. R. Volkas, Electroweak baryogenesis with vector-like leptons and scalar singlets, arXiv:1903.11255.

[69] S. Zheng, Minimal vectorlike model in supersymmetric unification, arXiv:1904.10145.

[70] CMS Collaboration, Search for vector-like leptons in multilepton final states in pp collisions at $\sqrt{s}=13 \mathrm{TeV}, \mathrm{CERN}$ Report No. CMS-PAS-EXO-18-005, 2018.

[71] A. M. Sirunyan et al. (CMS Collaboration), Search for vector-like leptons in multilepton final states in protonproton collisions at $\sqrt{s}=13 \mathrm{TeV}$, arXiv:1905.10853.

[72] Y. Cai, J. Herrero-Garcia, M. A. Schmidt, A. Vicente, and R. R. Volkas, From the trees to the forest: A review of radiative neutrino mass models, Front. Phys. 5, 63 (2017).

[73] S. Pascoli, R. Ruiz, and C. Weiland, Heavy neutrinos with dynamic jet vetoes: Multilepton searches at $\sqrt{s}=14,27$, and $100 \mathrm{TeV}$, J. High Energy Phys. 06 (2019) 049.

[74] P. Achard et al. (L3 Collaboration), Search for heavy neutral and charged leptons in $e^{+} e^{-}$annihilation at LEP, Phys. Lett. B 517, 75 (2001).

[75] M. L. Mangano et al., Physics at a $100 \mathrm{TeV}$ pp collider: Standard Model processes, CERN Yellow Report No. 3, 1, 2017; T. Golling et al., Physics at a $100 \mathrm{TeV}$ pp collider: 
Beyond the Standard Model phenomena, CERN Yellow Report No. 3, 441, 2017; R. Contino et al., Physics at a $100 \mathrm{TeV}$ pp collider: Higgs and EW symmetry breaking studies, CERN Yellow Report No. 3, 255, 2017; M. Mangano, Physics at the FCC-hh, a $100 \mathrm{TeV}$ pp collider, CERN Yellow Report No. CERN 2017-003-M, 2007.

[76] CEPC-SPPC Study Group, CEPC-SPPC preliminary conceptual design report. 1. Physics and detector, Reports No. IHEP-CEPC-DR-2015-01, No. IHEP-TH-2015-01, and No. IHEP-EP-2015-01; CEPC-SPPC preliminary conceptual design report. 2. Accelerator, Reports No. IHEPCEPC-DR-2015-01 and No. IHEP-AC-2015-01.

[77] X. Cid Vidal et al., Beyond the Standard model physics at the HL-LHC and HE-LHC, arXiv:1812.07831.

[78] T.-p. Li and Y.-q. Ma, Analysis methods for results in gamma-ray astronomy, Astrophys. J. 272, 317 (1983).

[79] R. D. Cousins, J. T. Linnemann, and J. Tucker, Evaluation of three methods for calculating statistical significance when incorporating a systematic uncertainty into a test of the background-only hypothesis for a Poisson process, Nucl. Instrum. Methods Phys. Res., Sect. A 595, 480 (2008).

[80] G. Cowan, K. Cranmer, E. Gross, and O. Vitells, Asymptotic formulae for likelihood-based tests of new physics, Eur. Phys. J. C 71, 1554 (2011).

[81] G. Cowan, Two Developments in Tests for Discovery: Use of Weighted Monte Carlo Events and an Improved Measure, Progress on Statistical Issues in Searches, SLAC (2012).

[82] M. Aaboud et al. (ATLAS Collaboration), Search for heavy charged long-lived particles in the ATLAS detector in $31.6 \mathrm{fb}^{-1}$ of proton-proton collision data at $\sqrt{s}=13 \mathrm{TeV}$, Phys. Rev. D 99, 092007 (2019).
[83] A. Alloul, N. D. Christensen, C. Degrande, C. Duhr, and B. Fuks, FEYNRULES 2.0-A complete toolbox for tree-level phenomenology, Comput. Phys. Commun. 185, 2250 (2014).

[84] J. Alwall, M. Herquet, F. Maltoni, O. Mattelaer, and T. Stelzer, MADGRAPH5: Going beyond, J. High Energy Phys. 06 (2011) 128.

[85] R. D. Ball, V. Bertone, S. Carrazza, L. Del Debbio, S. Forte, A. Guffanti, N. P. Hartland, and J. Rojo (NNPDF Collaboration), Parton distributions with QED corrections, Nucl. Phys. B877, 290 (2013).

[86] T. Sjostrand, S. Mrenna, and P.Z. Skands, PYTHIA 6.4 Physics and manual, J. High Energy Phys. 05 (2006) 026.

[87] T. Sjostrand, S. Ask, J. R. Christiansen, R. Corke, N. Desai, P. Ilten, S. Mrenna, S. Prestel, C. O. Rasmussen, and P. Z. Skands, An introduction to PYTHIA 8.2, Comput. Phys. Commun. 191, 159 (2015).

[88] J. de Favereau, C. Delaere, P. Demin, A. Giammanco, V. Lemaître, A. Mertens, and M. Selvaggi (DELPHES 3 Collaboration), DELPHES 3, A modular framework for fast simulation of a generic collider experiment, J. High Energy Phys. 02 (2014) 057.

[89] A. Djouadi, J. Kalinowski, and M. Spira, HDECAY: A program for Higgs boson decays in the standard model and its supersymmetric extension, Comput. Phys. Commun. 108, 56 (1998).

[90] H. K. Dreiner, H.E. Haber, and S. P. Martin, Twocomponent spinor techniques and Feynman rules for quantum field theory and supersymmetry, Phys. Rep. 494, 1 (2010).

[91] S.P. Martin, TASI 2011 lectures notes: Two-component fermion notation and supersymmetry, arXiv:1205.4076. 